\title{
TOWARDS A DEFINITION OF QUANTUM INTEGRABILITY
}

\author{
JESÚS CLEMENTE-GALLARDO AND GIUSEPPE MARMO
}

\begin{abstract}
We briefly review the most relevant aspects of complete integrability for classical systems and identify those aspects which should be present in a definition of quantum integrability.

We show that a naive extension of classical concepts to the quantum framework would not work because all infinite dimensional Hilbert spaces are unitarily isomorphic and, as a consequence, it would not be easy to define degrees of freedom. We argue that a geometrical formulation of quantum mechanics might provide a way out.
\end{abstract}

PACS:03.65.Ca, 02.40.Yy, 02.30.Ik

\section{INTRODUCTION}

In classical Mechanics, completely integrable systems have been widely studied in the past thirty years, many aspects have been considered and several approaches have been proposed $(22,23,54,36)$. Many soliton equations have been shown to admit a Hamiltonian formulation and to be completely integrable. Many completely integrable systems have been shown to arise as reduction of "simple" systems, very often associated with geodetical motions on Lie groups (46]). In this respect, they have been shown to be associated with Lie-Scheffers systems 35, 9, i.e. systems whose solutions admit a superposition rule [9. Thus we may safely say that classical completely integrable systems are well understood. On the other hand, it is commonly accepted that our description of the physical world should rely on Quantum Mechanics. As Dirac puts it: "classical mechanics must be a suitable limit of quantum mechanics".

Inspired by this statement, it is quite natural to look for a definition of quantum integrability whose appropriate limit would reproduce the classical definition and the multifacets situations one knows for classical systems, namely: multiHamiltonian descriptions, recursion operators, Lax pairs and so on 24, 34, 25. On the mathematical side, the quantization of soliton models has given rise to the beautiful structure of Yang-Baxter relations and the related formulation of quantum groups.

However, there are several problems if one tries to implement in a naive way the classical situation. Roughly speaking, the problems can be reduced to the fact that in infinite dimension all Hilbert spaces are isometrically isomorphic and therefore all $\mathbb{C}^{*}$-algebras of bounded operators acting on them are isomorphic. In particular, the idea of using the maximal set of commuting operators to imitate a maximal set of commuting first integrals of the classical situation does not work. The reason is a theorem by von Neumann stating that any two commuting operators can be written as functions of a third one, i.e. out of the finite dimensional maximal set of commuting operators we can find just one operator allowing us to express all the others as functions of it ([55]). 
From this point of view, what is missing is a way to distinguish, say, the Hilbert space of states of one particle from the Hilbert space of the states of many particles. In other terms: in the quantum setting it is not clear how to define the notion of degrees of freedom. Kirillov [29], for instance, suggested the idea of introducing the notion of "functional dimension". In some sense, we need to implement at the quantum level the notion of "functionally independent first integrals".

Besides these problems, other problems arise from the need of a sound definition of "suitable classical limit", i.e. to attach a mathematically reasonable meaning to the physicist's limit $\hbar \rightarrow 0$ [43].

At the moment, the way we consider this limit depends on the quantum picture we deal with. For instance, the Schrödinger picture versus the Heisenberg one, the first dealing with equations of motion on a Hilbert space the second dealing with equations of motion on a $\mathbb{C}^{*}$-algebra. The two pictures are usually connected by the so called $G N S$-construction [17. At the classical level the first picture corresponds to describe the dynamics as a symplectic vector field on a symplectic manifold, while the second one would correspond to the description of the dynamics as a Poisson derivation of a Poisson algebra.

By using this analogy we may consider the Hermitian structure on the vector space of states as the mathematical structure corresponding to the classical symplectic structure and similarly the Lie-Jordan algebra structure on observables would replace the classical Poisson structure.

However, before entering more deeply into the analysis of these analogies, let us consider what would be a reasonable approach to the integrability of a quantum evolution equation. Given the operator $H$ in some concrete realization, say the Schrödinger picture, we look for a Lie algebra $\mathfrak{g}$ and a group $G$ that are "naturally" associated with $H$. Typically, this would mean that there is a unitary representation of $G$ on the Hilbert space (the carrier space of our system) such that $H$ can be identified with the action of an element in the universal enveloping algebra $U(\mathfrak{g})$ of the Lie algebra $\mathfrak{g}$. The structure of the unitary representations of $G$ and their decomposition into irreducible ones then yields information about the spectrum and the eigenfunctions of $H$. This approach relies on the implicit assumption that the theory of unitary representations of $G$ is sufficiently developed so that it may be used as a non-commutative Fourier analysis (non-commutative Harmonic analysis on $G$ ). Nowadays a lot is known for a large class of connected finite-dimensional Lie groups ([53]).

Fundamental tools for this analysis are provided by Mackey's imprimitivity theory, a generalization of techniques elaborated by Weyl and von Neumann within quantum mechanics characterizing such representations; and the Kirillov-Konstant correspondence between representations and coadjoint orbits. For the use of Liealgebraic methods in the study of representations it is instrumental also the use of the space of smooth vectors (this originated with coherent states introduced by Schrödinger and further elaborated by Bargmann and Nelson); which is a module for the associative algebra $U(\mathfrak{g})$.

The connection with biHamiltonian classical systems arises with the introduction of biunitary operators. They are associated with one-parameter groups of transformations which are unitary for alternative Hermitian structures on the same topological vector space of states $([40,43])$. The geometrical formulation of Quantum Mechanics turns out to be very useful to identify the relevant mathematical 
structures so that we may, more easily formulate what we mean by alternative structures compatible with a quantum evolution.

Let us recall briefly the fundamental aspects of classical integrability.

\section{Composition OF MOdel DYNAMiCS}

2.1. Classical integrability. From a classical point of view, the theory of integrable systems is very well established. In this section we shall just discuss a few of the best known properties which will become later important when compared to the analogue situation in the quantum framework.

Let us begin considering the usual definition of a classical integrable system. Consider a classical system with $n$ degrees of freedom, defined on some differential manifold $M$. For simplicity, let us consider a dynamics $\Gamma$ defined via a Hamiltonian function $H$ on the cotangent bundle $T^{*} M$. We will consider the canonical Poisson structure associated to the canonical symplectic form, and the corresponding Poisson bracket defined on $C^{\infty}\left(T^{*} M\right)$.

Definition 1. The system is said to be integrable if there exists

- a set of $n$ functions $\left\{f_{j}\right\}_{j=1}^{n}$, where $f_{k} \in C^{\infty}\left(T^{*} M\right)$, which are constants of the motion

$$
0=\left\{H, f_{k}\right\} \quad \forall k
$$

- functionally independent $d f_{1} \wedge \cdots \wedge d f_{n} \neq 0$

- which are in involution, i.e.

$$
\left\{f_{k}, f_{j}\right\}=0 \quad \forall j, k
$$

Of course we could consider more general situations to describe systems whose dynamics can be explicitly integrated. In principle, just the flow of the system on the given manifold is to be considered as the object of interest. The geometrical structures around it may be changed and actually this leads to important consequences in some situations, as in the case of biHamiltonian systems.

There a few properties of these systems which we would like to consider more closely. First of all, one of the most relevant properties is that the dynamics for such a system is always integrable by quadratures. For instance, we can write the dynamics in a trivial way in a new set of coordinates, defined by the set of the constants of the motion and their corresponding conjugate variables. These can always be found, at least locally [3, 1, 41]:

Theorem 1. There exists a set of coordinates $\left\{I_{j}, \phi_{j}\right\}_{j=1}^{n}$ such that the mapping $\Phi:\left(q^{i}, p_{i}\right) \mapsto\left(I_{k}, \phi_{k}\right)$ is a local symplectomorphism and such that the vector field $\Phi_{*}(\Gamma)$ is written as

$$
\Phi_{*}(\Gamma)=\sum_{k} \omega_{k}(I) \frac{\partial}{\partial \phi_{k}}
$$

This is one of the relevant features we would like to be able to recover at the quantum level: the possibility of integrating completely the dynamics in a constructive way. This is one of the more involved points in what regards the definition of quantum integrability, because, within the quantum setting, it requires nonlinear transformations of non commuting variables, and we shall discuss it briefly.

There is another aspect of classical integrability which we would like also to mention here. The definition above is somehow restrictive from certain points 
of view. If what we want is just to integrate the dynamics, we could drop the requirement that $\Phi$ be a symplectomorphism and just require that (1) is a vector field which is nilpotent of index two. This would allow to cover much more general situations. Let us review this quickly:

2.2. The meaning of Arnold-Liouville theorem. From our point of view, Arnold-Liouville's theorem (AL in the following) can also be read in a different way. One should interpret it as a way of relating a given system, defined in the original manifold $M$ with a set of systems defined on suitable associated model manifolds $\left\{N_{j}\right\}$ via "projections" $\Phi_{j}: M \rightarrow N_{j}$, defined in action-angle variables, whose dynamics is trivially integrable. The role of the $n$ constants of the motion in involution is just to give rise to the transformation which allow to define these "projections". This transformation is nothing but the set of mappings defining each torus as a leaf of the foliation of $M$ according to the level sets of the momentum mapping. Each leaf may be therefore identified with our manifold $N_{j}$, and the transformation

$$
\left\{\left(q_{i}, p^{i}\right)\right\} \mapsto\left(I_{j}, \phi_{j}\right)
$$

is the corresponding mapping $\Phi_{j}$.

The aspect that we want to point out is that, from this perspective, AL construction is analogous to the transformation of a linear system into Jordan blocks. The submanifolds characterized by $\Phi$ and $\nu$ are now the analogues of the Jordan blocks. There is an important difference however: AL theorem shows that by using nonlinear canonical transformations we can relate generically any complete integrable systems with two types of models:

- "Harmonic oscillator"-type for those systems whose dynamics is completely bounded

- "free particles"-type for those systems with unbounded motions.

The decomposition of the dynamical vector field is also obtained as above. Suppose that we are working with action-angle variables on the classical manifold $M$ that we assume to be symplectic. Classical dynamics is described in terms of a vector field, which is the derivation associated to the classical Hamiltonian $H$. Suppose we have an integrable system, whose Hamiltonian vector field is written, in the usual way, in action-angle variables $\left\{I_{j}, \phi_{j}\right\}_{j=1 \cdots n}$ as:

$$
X_{h}=\nu_{i} \frac{\partial}{\partial \phi^{i}}
$$

where each $\nu_{j} \in \mathbb{R}$ is the corresponding "frequency" and we assume $\phi_{j} \in(-\pi, \pi)$ (we assume hence each single dynamics will be bounded). This vector field describes the motion of a "classical Harmonic oscillator" and its integral curves are contained in the corresponding toroidal manifold which serves as level set for the momentum mapping. On any torus, the dynamical vector field has the same form, since the frequencies are constant, however, in general, these constants may depend on the torus

\section{Remark}

We should point out that by means of a nonlinear transformation we are going from a system described, say, by the matrix

$$
\left(\begin{array}{cc}
0 & -1 \\
1 & 0
\end{array}\right)
$$


in the $(q, p)$ coordinates to a system described by the nilpotent matrix

$$
\left(\begin{array}{ll}
0 & 1 \\
0 & 0
\end{array}\right)
$$

in the $(\Phi, \nu)$ coordinates. Thus we are considering a nonlinear transformation which relates two linear dynamical systems described by completely different matrices.

We may also describe the structure above in a generalized fashion by requiring the existence of a set of functionally independent functions $\left\{J_{1}, \cdots, J_{2 n}\right\}$ which satisfy

$$
\mathcal{L}_{\Gamma}\left(\mathcal{L}_{\Gamma} J_{j}\right)=0,
$$

with $\Gamma$ being the dynamical vector field. We obtain in this way a description in terms of generalized "action-angle functions", which need not be pairwise "conjugate", instead of the local "action-angle coordinates". It means, in particular, that a completely integrable system is nilpotent of order two, on some set of functionally independent functions which separate points of the carrier space. In these "coordinate functions" the formal exponentiation gives

$$
e^{t L_{\Gamma}} J_{j}=J_{j}+t L_{\Gamma} J_{j},
$$

i.e. the motion is linear in time. If we replace $J_{j}$ with $\left(I_{j}, \phi_{j}\right)$ we recover the dynamics on each torus:

$$
I_{j}(t)=I_{j}(0) \quad \phi_{j}(t)=\phi_{j}(0)+t \nu_{j}
$$

Let us consider more closely integrable systems which are not described by isoperiodic motions. The vector field on each torus is always of the same type as above, but now the frequencies exhibit a dependence on the action variables, i.e. $\nu_{i}=\nu_{i}\left(I_{1}, \cdots, I_{n}\right)$. On each torus the vector field is "equivalent" to the vector field of the Harmonic oscillator, i.e. a linear combination of the angular vector fields, but the coefficients are different for each torus. As a consequence, we can say that a classical integrable system is made up by a suitable combination of vector fields corresponding to Harmonic oscillators, where suitable means that the oscillator vector fields from each toroidal leaf are "composed" by using "coupling constant" which are functions of the action variables.

2.3. Conjugate systems and classical integrability. In this section we want to elaborate further, with some additional details and from a more geometrical approach, the interpretation of AL theorem that we just described. The idea is that the integrability of a vector field $X$ (the dynamical vector field of our system) may be related with that of a set of models $\left\{Y_{j}\right\}$, related to a transversal decomposition of the original manifold, whose evolution is well known. So we say that the integrability of each one of the $Y_{j}$ ensures the integrability of the vector field $X$, which is supposed to be decomposed in an analogous manner to the canonical "Jordan form" of a linear system. We will see in the following that this procedure offers many advantages.

A crucial part of the present work concerns thus the relation between structures belonging to different spaces, particularly vector fields and algebras of functions. We will begin with the differential geometric preliminaries needed for the understanding of our considerations.

Let us consider two differential manifolds, $M$ and $N$ and a differentiable mapping between them $\Phi: M \rightarrow N$ We suppose that we have a vector field on each manifold (the dynamical vector field for instance), and we want that $\Phi$ relates the different 
set of orbits on each space, in a 1:1 correspondence, without requiring that they have the same parametrization. The general expression for this correspondence is summarized in the expression:

$$
T \Phi \circ X=f Y \circ \Phi
$$

where $T \Phi$ is the induced tangent bundle map acting on vector fields, i.e. sections of $T M$ and $T N$ respectively, and $f$ is an arbitrary function on $N$. In other words, $X$ and $Y$ are $(\Phi, f)$-related, i.e. $X$ and $f Y$ are $\Phi$-related (this is a way to formalize the statement that the orbits are in one-to-one correspondence up to parametrization).

When this mapping is such that $f=1$ we have a 1:1 correspondence not only for orbits, but also for integral curves, i.e. the image by $\Phi$ of any integral curve of $X$ on $M$ is an integral curve of $Y$ on $N$; in this case we say simply that $X$ and $Y$ are $\Phi$-related.

We will use now this kind of mappings, suitably combined, to decompose the dynamics on a given manifold, defined through a Hamiltonian function or a Hamiltonian vector field, in a set of well known dynamics on special manifolds which we will call "models".

In fact we will not use the dynamics on the full manifold but just on an open dense submanifold, extending suitably.

Let us apply now some general considerations. Let $M$ be a symplectic manifold (it is not necessary, but it will be so for our applications) and let $X \in \mathfrak{X}(M)$ be the dynamical vector field. Let us call model systems $\mathcal{M}_{j}$, some pairs $\left(Y_{j}, N_{j}\right)$ where $N_{j}$ is a differential manifold and $Y_{j} \in \mathfrak{X}\left(N_{j}\right)$. We suppose we can define a covering $\left\{M_{j}\right\}$ of $M$ along with a set of diffeomorphisms:

$$
\Phi_{j}: M_{j} \subset M \rightarrow N_{j}
$$

which posses property (2).

Now we want to use these sets $N_{j}$ and these vector fields $Y_{j}$ to describe the dynamics corresponding to $X$. We can choose two different ways for doing it: working at the level of vector fields and manifolds or working at the level of functions and their algebraic structures:

a) We can work at the level of vector fields, where we know the dynamics is described by $X$, and we can try to find a suitable set of vector fields $Y_{j}$ in such a way that we can integrate this dynamics with their aid. We suppose that $(2)$ holds for any $N_{j}$, and that a decomposition may be chosen in such a way that we could write:

$$
X=\sum_{j} h_{j} \widetilde{Y}_{j}
$$

where $\mathcal{L}_{Y_{i}} h_{j}=0 \quad \forall i \neq j$ and $\widetilde{Y}_{j} \in \mathfrak{X}(M)$ is the vector field on $M \Phi_{j}$-related with $Y_{j}$.

In this set, we define:

Definition 2. We say that the model set $\mathcal{M}=\left\{\left(N_{j}, Y_{j}\right)\right\}$ gives a transversal decomposition for $\mathfrak{X}(M)$ when we can write:

$$
\mathfrak{X}(M)(m)=\bigoplus_{j}\left[\mathfrak{X}_{j}^{\uparrow}\left(N_{j}\right)\right](m)
$$


which means that at any point of the manifold $M$ the "lifts" of the vectors fields from $N_{j}$ to $M$ define a basis of the corresponding tangent space $T_{m} M$.

Thinking in terms of the $C^{\infty}(M)$-module structure of the set of vector fields of $M$, it is also interesting to further analyze this decomposition. We could say that the set of vector fields $\left\{\widetilde{Y}_{j}\right\}$, the lifted vector fields, defines a system of generators for the module $\mathfrak{X}(M)$ with coefficients in $C^{\infty}(M)$.

When we work in classical dynamics, the physical observables are functions $C^{\infty}(M)$, and therefore we want to describe the previous construction in terms of functions. We can restate the previous requirements in the form

$$
\mathcal{L}_{\widetilde{Y}_{j}} \Phi_{j}^{*}(f)=\Phi_{j}^{*}\left(L_{Y_{j}} f\right) \quad \forall f \in C^{\infty}\left(N_{j}\right) .
$$

b) We can also work at the level of functions on each manifold with the algebra structure provided by the Poisson bracket. The dynamics now is described with the Hamiltonian function and its adjoint action inside the Poisson bracket:

$$
\frac{d f}{d t}=\{f, h\}
$$

The set of models now is just specified by $\left\{N_{j}\right\}$ (and the corresponding mappings $\Phi_{j}$ ), since we write the definition of transversal decomposition as:

Definition 3. Given $M$ and $X$ as above, and the set of models and mappings $\left(N_{j}, \Phi_{j}\right)$ we say that they define a transversal decomposition of an open dense submanifold of $M$ when the set $\bigoplus_{j} d \Phi_{j}^{*}\left(C^{\infty}\left(N_{j}\right)\right)$ generates $\mathfrak{X}^{*}(M)$ as a module (the set of 1-forms on $M$ ). As above we are requiring that

$$
d \Phi_{j}^{*}\left(C^{\infty}\left(N_{j}\right)\right) \cap d \Phi_{i}^{*}\left(C^{\infty}\left(N_{i}\right)\right)=0, \quad i \neq j .
$$

And moreover, we say that a vector field $X \in \mathfrak{X}(M)$ is decomposable according to the set of models $\left\{\Phi_{j}\right\}$ if

$$
\mathcal{L}_{X} \Phi_{j}^{*}\left(C^{\infty}\left(N_{j}\right)\right) \subset \Phi_{j}^{*}\left(C^{\infty}\left(N_{j}\right)\right)
$$

i.e. each subring $\Phi^{*}\left(C^{\infty}\left(N_{j}\right)\right)$ of functions is stable under the action of the dynamical vector field.

It is important to remark that both approaches are not trivially related, since the set of Hamiltonian vector fields (which is the set arising in this latter one) does not define a $C^{\infty}(M)$-module, and therefore there is no possible transversal decomposition associated with them.

\section{Remark}

From this point of view, even non-Hamiltonian systems may be considered integrable. In the first cases we have discussed objects corresponding to the infinite dimensional Lie algebra of Hamiltonian vector fields. But it is also possible to formulate things directly at the level of the $C^{\infty}(M)$-module of vector fields as a whole. Suppose that the dynamical field can be written as a linear combination of derivations that are inner when restricted to a torus, with coefficients depending on the action variables. Using the preceding formalism we say that in the combination above we do not need to impose that the functions $h_{j}$ belong to $\Phi_{j}^{*}\left(N_{j}\right)$ but just that:

$$
\mathcal{L}_{\widetilde{Y}_{j}} h=0
$$


and therefore the derivations corresponding to $X$ need not be inner (corresponding to a Hamiltonian on $M$ ) on the whole manifold, but that it coincides on each torus with an inner derivation which may depend on the torus. We could have a function $h_{j}=h_{j}\left(I_{1}, \cdots, I_{n}\right)$ which is constant on each torus, but that depends on all of the constants of the motion, the equation (4) above implies only that the Hamiltonian commutes with the function $h_{j}$ and therefore its most general form is an arbitrary function of the constants of the motion of the system.

To put it differently, we could use one-forms which are not closed to describe vector fields by means of the symplectic structure. What we require is only that the one-form is expressed in terms of action-angle variables. In different terms, in the equations

$$
\dot{I}^{k}=0 \quad \dot{\phi}^{k}=\nu^{k}\left(I_{1}, \cdots, I_{n}\right),
$$

we may drop the requirement that $\nu^{k}$ are partial derivatives of a function (the Hamiltonian function) [2].

2.4. Examples of classical integrable systems. Now we are able to give a few examples which illustrate the situation in the classical case. We can consider two families:

(1) Systems whose orbits are on tori. Model systems in this case would be just combinations of one dimensional Harmonic oscillator. Examples of this group would be:

- The $n$ dimensional Harmonic oscillator

- The $q$-deformed oscillator, which would be the simplest non trivial example.

- The closed orbits of the Kepler problem.

(2) Systems whose orbits are on cylinders (i.e. on $\mathbb{R}^{k} \times T^{n-k}$ ). The model systems now have to combine the one dimensional Harmonic oscillator and the free particle, to include the unbounded motion. As examples of this case we could mention:

- The unbounded orbits of the Kepler problem.

- The Calogero-Moser type potentials.

2.4.1. Example: the Harmonic oscillator. Let us develop now some of these examples. Let us begin with systems defined on tori.

For this kind of systems, the preceding description of Liouville integrability is straightforwardly adapted. Let us take as a first example the $n$ dimensional Harmonic oscillator:

$$
H=\sum_{k} \frac{1}{2}\left(p_{k}^{2}+\omega_{k} q_{k}^{2}\right)
$$

If we select as first integrals the partial energies, we can define:

$$
I_{j}=h_{j}=\frac{1}{2}\left(p_{j}^{2}+\omega_{j} q_{j}^{2}\right)
$$

and the Hamiltonian is therefore written:

$$
H=\sum_{k} h_{k}=\sum_{k} I_{k}
$$


Liouville-Arnold theorem provides us also with the angle variables, and the conformal factor $F_{k}$ is obtained following the expression above as:

$$
f_{k}=\frac{\partial H}{\partial I_{k}}=\omega_{k}
$$

Obviously, the Harmonic oscillator turns out to be integrable, and its dynamics linear.

The following example is the first non trivial modification: the $q$-deformed classical oscillators that can be found for instance in [39. The Hamiltonian may be written as:

$$
H=\omega \sum_{k} \frac{\sinh \hbar \alpha_{k} \alpha_{k}^{*}}{\sinh \hbar}
$$

where $\alpha_{k}=\frac{1}{\sqrt{2}}\left(\frac{i p_{k}}{\sqrt{\omega}}+\sqrt{\omega} q_{k}\right)$.

The invariant now is the classical "number operator", but the conformal factor will not be a numerical constant, but a constant of the motion, i.e. a function of the invariants:

$$
\begin{gathered}
I_{k}=\alpha_{k} \alpha_{k}^{*} \\
f_{k}=\frac{\partial H}{\partial I_{k}}=\omega \frac{\hbar}{\sinh \hbar} \cosh \hbar \alpha_{k} \alpha_{k}^{*}
\end{gathered}
$$

The role played by this conformal factor is quite clear, it represents a frequency that is not constant on the phase space, but it is constant on each manifold $I_{k}, \phi_{k}$.

2.4.2. Conformal factors. On $\mathbb{R}^{2}$, with coordinates $(x, p)$ we consider the dynamical system:

$$
\dot{x}=p f\left(x^{2}+p^{2}\right) \quad \dot{p}=-x f\left(x^{2}+p^{2}\right)
$$

It is immediate that the orbits of this system are all circles with center at the origin. We can integrate our system for any initial condition $\left(x_{0}, p_{0}\right)$ :

$$
\left(\begin{array}{l}
x(t) \\
p(t)
\end{array}\right)=\exp \left[t f\left(x_{0}^{2}+p_{0}^{2}\right)\left(\begin{array}{cc}
0 & 1 \\
-1 & 0
\end{array}\right)\right]\left(\begin{array}{l}
x_{0} \\
p_{0}
\end{array}\right)
$$

It is clear that we are using the Harmonic oscillator system

$$
\begin{cases}\dot{x} & =\omega p \\ \dot{p} & =-\omega x\end{cases}
$$

to find our flow and replace the constant frequency $\omega$ with a constant of the motion $f$. If, however, we look for a diffeomorphism "conjugating" our starting system with the Harmonic oscillator, we would find that no such a diffeomorphism can exist because we know that the period of each orbit is invariant under diffeomorphisms, and our two systems have different periods for each value of the radius. Therefore the conformal factor will help in declaring our system "conformally equivalent" to the Harmonic oscillator. Thus without the conformal factor, each $f$ would provide a different equivalence class. 
2.4.3. The Kepler problem. We consider now the case of the Kepler problem. In principle the idea is simple, we have just to proceed as we did above. As a first point, the action-angle variable have to be determined. Following [4] we find that:

$$
\begin{aligned}
J_{1}= & -\sqrt{p_{\theta}^{2}+\frac{P_{\phi}^{2}}{\sin ^{2} \theta}}+m k\left(\frac{2 m k}{r}-\frac{p_{\theta}^{2}}{r^{2}}-\frac{p_{\phi}^{2}}{r^{2} \sin ^{2} \theta}-p_{r}^{2}\right)^{-1} \\
J_{2}= & \sqrt{p_{\theta}^{2}+\frac{P_{\phi}^{2}}{\sin ^{2} \theta}-p_{\phi}} \\
J_{3}= & p_{\phi} \\
\phi_{1}= & -\frac{\sqrt{-m^{2} k^{2} r^{2}+2 m k\left(J_{1}+J_{2}+J_{3}\right)^{2} r-\left(J_{1}+J_{2}+J_{3}\right)^{2}\left(J_{2}+J_{3}\right)^{2}}}{\left(J_{1}+J_{2}+J_{3}\right)^{2}}+ \\
\phi_{2}= & \phi_{1}-\arcsin \frac{m k r-\left(J_{1}+J_{2}+J_{3}\right)^{2}}{\left(J_{1}+J_{2}+J_{3}\right) \sqrt{\left(J_{1}+j_{2}+J_{3}\right)^{2}-\left(J_{2}+J_{3}\right)^{2}}} \\
\phi_{3}= & \phi_{2}+\arcsin \frac{J_{3} \cot \theta}{\left.\sqrt{\left(J_{1}+j_{2}+J_{3}\right)^{2}-\left(J_{2}+J_{3}\right)^{2}}-\arcsin \frac{\left(J_{3} J_{3}^{2}\right.}{\sqrt{\left(J_{2}\right.}}+J_{3}\right) \cos \theta}(10)
\end{aligned}
$$

Analogously, we can write the Hamiltonian in terms of these variables:

$$
H=-\frac{m k^{2}}{\left(J_{1}+J_{2}+J_{3}\right)^{2}}
$$

From our previous discussion, we can compute now the conformal factor for this case, simply as the derivative:

$$
f_{k}=\frac{\partial H}{\partial J_{k}}
$$

For this case there is just one conformal factor:

$$
f_{1}=f_{2}=f_{3}=\frac{2 m k^{2}}{\left(J_{1}+J_{2}+J_{3}\right)^{3}},
$$

and the related vector field acquires the form:

$$
X=\frac{2 m k^{2}}{\left(J_{1}+J_{2}+J_{3}\right)^{3}}\left(\frac{\partial}{\partial \phi_{1}}+\frac{\partial}{\partial \phi_{2}}+\frac{\partial}{\partial \phi_{3}}\right)
$$

Now we have to consider how to extend these structures to the quantum domain. It is clear that the geometrical formulation of Quantum Mechanics allowing for nonlinear transformations on the space of pure states, the space of rays or the complex projective space, will be more appropriate to carry on our considerations.

\section{The Problems of Quantum integrability}

Let us try to adapt to the quantum setting the results of classical integrability we have analyzed. We recall that in order to be able to explicitly integrate the system, the main properties and requirements of $\mathrm{AL}$ theorem were

- the number of degrees of freedom of the system, which determines the dimension of the manifold where the system evolves, is equal to the number of functionally independent constants of the motion in involution. 
- it is important to characterize the geometrical objects the system may be associated with. The reason for this is that the existence of different geometrical structures compatible with a given dynamics may provide new constants of the motion (as it happens with classical biHamiltonian systems, for instance)

- the notion of independence of the constants of the motion is crucial for them to be used as coordinates in the action-angle description (and therefore they must be functionally independent).

All these properties, well known and controlled in the classical framework, are not without ambiguities when transferred to the quantum setting. Let us study them in some detail:

3.1. The notion of degrees of freedom. Let us now turn our attention towards the von Neumann theorem we mentioned in the introduction. It reads as follows:

Theorem 2. Given a Hilbert space $\mathcal{H}$ and two observables $A, B$, if they commute, there exists a third operator $C$ such that both $A$ and $B$ are functions of $C$.

Thus if we look for observables which commute among themselves and with the Hamiltonian, as the naive generalization of AL theorem would suggest, we face the problem of dealing with their independence since the theorem we have quoted asserts that they will be functionally dependent on just one operator $C$. We can not then relay on the number of degrees of freedom of the system, for this is a concept which does not have a clear meaning in the usual quantum setting. Let us elaborate a bit in this direction.

We are going to see how it is not possible to define a reasonable notion of degree of freedom in the case of quantum systems. To this aim, we shall consider an example taken from a paper by Weigert [56] and further elaborated by Hietarinta 28.

Consider the case of a one-dimensional Harmonic oscillator

$$
H=\hbar \omega\left(a^{\dagger} a+\frac{1}{2}\right)=\hbar \omega\left(\hat{n}+\frac{1}{2}\right) .
$$

We recall that there is a countable set of eigenstates for the number operator $\hat{n}$ :

$$
\hat{n}|n\rangle=n|n\rangle \quad n \in \mathbb{N}_{0}=\{0,1,2, \cdots\}
$$

which constitute an orthonormal basis for the carrier Hilbert space of states of our system. For the creation and annihilation operators we have, in this basis,

$$
a|n\rangle=\sqrt{n}|n-1\rangle \quad a^{\dagger}|n\rangle=\sqrt{n+1}|n+1\rangle
$$

along with

$$
|n\rangle=\frac{\left(a^{\dagger}\right)^{n}}{\sqrt{n !}}|0\rangle .
$$

We would expect that this system, being one dimensional, should have only one constant of the motion. However, we are going to show how it is possible to define two constant of the motion in involution by using the following trick.

Let us replace the label $n$ by two indices $n_{1}$ and $n_{2}$, both taking all non-negative integer values and related to $n$ by the following relation

$$
n=n_{1}+\frac{1}{2}\left(n_{1}+n_{2}\right)\left(n_{1}+n_{2}+1\right) .
$$


We can list then a few correspondences:

$$
\begin{aligned}
n=0 \longrightarrow\left(n_{1}, n_{2}\right)=(0,0) \quad n=1 \longrightarrow\left(n_{1}, n_{2}\right)=(0,1) \quad n=2 \longrightarrow\left(n_{1}, n_{2}\right)=(1,0) \\
n=3 \longrightarrow\left(n_{1}, n_{2}\right)=(0,2) \quad n=4 \longrightarrow\left(n_{1}, n_{2}\right)=(1,1)
\end{aligned}
$$

Considering a two dimensional grid (in an analogous way we can repeat the argument with three operators getting thus a cubic grid, etc) we find the following diagram

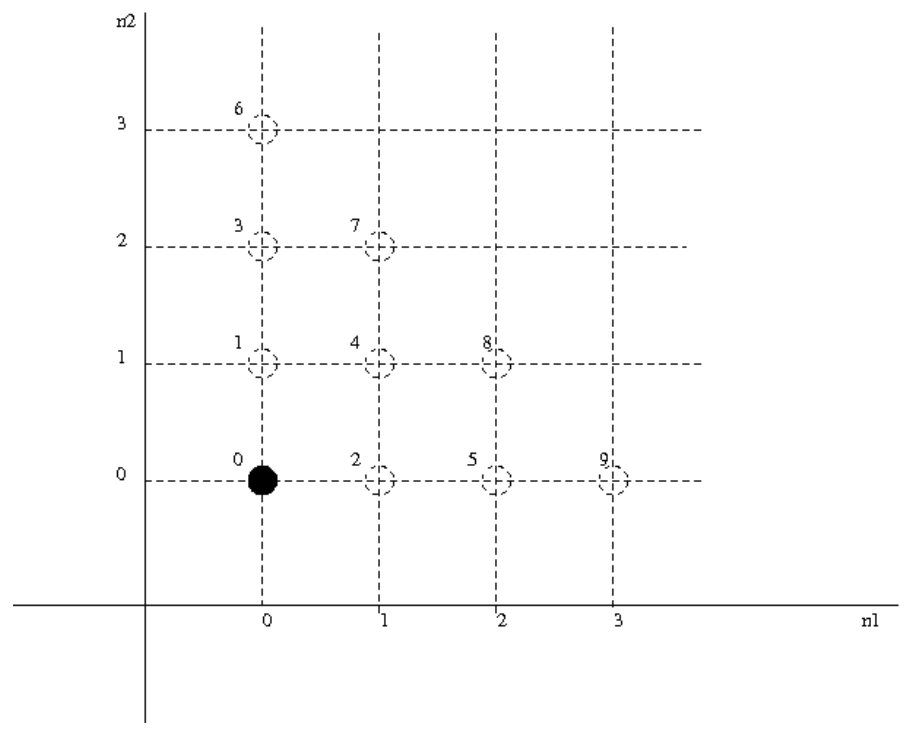

Every pair $\left(n_{1}, n_{2}\right)$ determines exactly one integer number $n$ and vice-versa. Therefore it is possible to label the states $|n\rangle$ by using the two integer-valued labels $\left(n_{1}, n_{2}\right)$ :

$$
|n\rangle \equiv\left|n_{1}, n_{2}\right\rangle
$$

We can find, obviously, an orthonormal basis

$$
\left\langle n_{1}, n_{2} \mid n_{1}^{\prime}, n_{2}^{\prime}\right\rangle=\delta_{n_{1}, n_{1}^{\prime}} \delta_{n_{2}, n_{2}^{\prime}},
$$

along with a decomposition of the identity

$$
\sum_{n_{1}, n_{2}}\left|n_{1}, n_{2}\right\rangle\left\langle n_{1}, n_{2}\left|=\sum_{n}\right| n\right\rangle\langle n|=\mathbb{I}
$$

These states can be considered as the eigenstates of two operators, $\hat{n}_{1}$ and $\hat{n}_{2}$, independent and hence commuting among themselves.

The eigenvalues of the Hamiltonian operator may be written as

$$
E_{n}=\hbar \omega\left(n_{1}+\frac{1}{2}\left(n_{1}+n_{2}\right)\left(n_{1}+n_{2}+1\right)+\frac{1}{2}\right) \quad n_{1}, n_{2} \in \mathbb{N}_{0}
$$

The states $|0\rangle,|1\rangle,|3\rangle, \cdots\left|\frac{1}{2} k(k+1)\right\rangle$ belong to $n_{1}=0$ and therefore the eigenvalue has infinite degeneracy. Similarly for $\hat{n}_{2}$, for which we can write:

$$
\hat{n}_{2}=\sum_{l=0} \sum_{k} l \hat{P}\left(l+\frac{1}{2}(k+l)(k+l+1) .\right.
$$

Here $\hat{P}(m)=|m\rangle\langle m|$, i.e. this represents the operator giving the spectral decomposition for $\hat{n}_{2}$. 
It is clear that $\left[\hat{n}_{1}, \hat{n}_{2}\right]=0$ and that the total Hamiltonian operator can be written as

Obviously,

$$
\hat{H}=\hbar \omega\left(\hat{n}_{1}+\frac{1}{2}\left(\hat{n}_{1}+\hat{n}_{2}\right)\left(\hat{n}_{1}+\hat{n}_{2}+1\right)+\frac{1}{2}\right) .
$$

But this means that

$$
\left[\hat{H}, \hat{n}_{1}\right]=0=\left[\hat{H}, \hat{n}_{2}\right]
$$

Lemma 1. The one dimensional Harmonic oscillator above has two constants of the motion commuting with each other.

We can consider the eigenstates $|n\rangle$ as belonging to the Hilbert space which is the tensor product $\mathcal{H}_{1} \otimes \mathcal{H}_{2}, \mathcal{H}_{i}$ being the Fock space corresponding to each number operator. Both operators $\hat{n}_{1}$ and $\hat{n}_{2}$ have infinite degeneracy, but the states of the composed operator $\hat{n}$ has none.

$$
\hat{H}|k\rangle=k|k\rangle \quad k \in \mathbb{N}_{0} \quad|k\rangle=\left|n_{1}\right\rangle \otimes\left|n_{2}\right\rangle \quad n_{1}, n_{2} \in N_{0} .
$$

This has been done for the particular case of the Harmonic oscillator, but as we are going to see, any one-dimensional quantum system (with discrete spectrum and eigenvalues bounded from below) can be given previous formulation. Consider for instance a system described by the Hamiltonian

$$
\frac{\hat{P}^{2}}{2 m}+\hat{V}(q)
$$

and assume its spectrum is lower bounded as $E_{0}<E_{1}<E_{2}<\cdots$. Denote the elements of the orthonormal basis of eigenstates as $\left\{\left|E_{i}\right\rangle\right\}_{i=0,1, \ldots}$.

We can define now linear operators on the complex Hilbert space spanned by the above vectors by setting:

$$
A\left|E_{k}\right\rangle=\sqrt{k}\left|E_{k-1}\right\rangle ; \quad A^{\dagger}\left|E_{k}\right\rangle=\sqrt{k+1}\left|E_{k+1}\right\rangle,
$$

along with

$$
A\left|E_{0}\right\rangle=0 ; \quad \frac{\left(A^{\dagger}\right)^{n}}{\sqrt{n !}}\left|E_{0}\right\rangle .
$$

It is now obvious that the product $A^{\dagger} A \equiv \hat{N}$ has the same eigenvectors as the original Hamiltonian $\hat{H}$ and thus $\hat{H}$ can be written as a function of $\hat{N}$. Therefore the same considerations we gave above for the Harmonic oscillator can be given for a generic Hamiltonian (with the properties on the spectrum we discussed above). But this implies that the system admits two constants of the motion $\hat{N}_{1}$ and $\hat{N}_{2}$, in terms of which the Hamiltonian can also be written.

More generally, given a maximal set of commuting operators, uniquely identifying an orthonormal basis for the Hilbert space, once we order these states by means of $\mathbb{N}_{0}$, we can define an operator

$$
\hat{K}=\sum_{k} \frac{|k\rangle\langle k|}{(k+1)^{2}} \quad k \in \mathbb{N}_{0}
$$

Thus, any element of the family of commuting operators will be an appropriate function of $\hat{K}$.

It is clear that these peculiarities are to be traced in the fact that all infinite dimensional Hilbert spaces are isomorphic, indeed unitarily isomorphic. If $|n\rangle_{(a)}$ 
(with $n \in \mathbb{N}$ ) provides an orthonormal basis for $\mathcal{H}_{(a)}$ (where $\left.a=1,2, \cdots\right)$, we can establish this correspondence by setting $|n\rangle_{2}=T|n\rangle_{1}$. That being so, any operator $A_{(1)}$ on $\mathcal{H}_{(1)}$ has a corresponding operator $A_{(2)}=V A_{(1)} V^{-1}$ on $\mathcal{H}_{(2)}$. Thus also the corresponding $\mathbb{C}^{*}$-algebras are isomorphic. This means that, at an abstract level, we can not implement a notion of degree of freedom, because the Hilbert space of one particle is isomorphic with the Hilbert space of many particles (Balachandran: bringing up a quantum baby).

With these examples we hope to have convinced the reader that the notion of degree of freedom in the framework of Quantum Mechanics does not have, at the moment, a sound mathematical definition.

3.2. The integration of the dynamics. From the considerations we have made in Section 3.1. it is clear that we need a notion of functional independence for differentiable functions to be able to distinguish the carrier space of one particle from the carrier space of many particles.

To the extent that the differential calculus is developed nowadays, to deal with (local) derivations we need commutative algebras. If we start with algebras of operators on some Hilbert space, the spectral theorem asserts that every Hermitian operator is unitarily equivalent to a multiplication function (operator).

Thus, out of a family of commuting Hermitian operators we get a family of multiplication functions. According to the Gelfand-Naimark theory, the point spectrum of these commutative functions, with some additional requirements, can be given the structure of a smooth manifold. This seems to be the main route to extract a "classical manifold" from a quantum carrier space. (As a matter of fact, the theory provides us with a Hausdorff topological space; to get a smooth manifold one has to select a subset of states, often $C^{\infty}$ or analytic vectors) [15, 31, 48.

In some other approaches these aspects may be hidden in other assumptions and they appear in disguise. In many instances they appear very openly because the Hilbert spaces are required to be concretely realized as square integrable functions on some measure space, the algebra of operators is identified from the beginning with differential operators (in this case the commutative algebra of multiplications functions i.e. differential operators of degree zero, is immediately identified). A further choice of a specific quantum system, i.e. a specific Hamiltonian operator with a corresponding domain of self-adjointness, will select further topological and differential structures of the "classical manifold".

In what follows we make few considerations on a procedure which subsumes most of the existing approaches to quantum integrability. We consider the equations of motion on some Hilbert space $\mathcal{H}$, in the Schrödinger picture

$$
i \hbar \frac{d}{d t} \psi=H \psi
$$

or with the equations of motion on the algebra of operators (Heisenberg picture):

$$
i \hbar \frac{d}{d t} A=[H, A]
$$

We look for a Lie algebra $\mathfrak{g}$ and a related Lie group $G$ that are "naturally" associated with $H$.

Typically, we look for a unitary representation of $G$ in the Hilbert space $\mathcal{H}$ and require that the Hamiltonian operator can be identified with the action of an element in the universal enveloping algebra $U(\mathfrak{g}), \mathfrak{g}$ being the Lie algebra of $G$. 
The structure of the unitary representations of $G$ and their decomposition in irreducible ones yields information about the spectrum and the eigenfunctions of $H$. Let us try to be more specific by recalling a few facts about representation theory:

A unitary representation of $G$ in the Hilbert space $\mathcal{H}$ is a homomorphism $\pi$ of $G$ into the group of unitary transformations $U(\mathcal{H})$ with the following continuity condition:

The map $G \times \mathcal{H} \rightarrow \mathcal{H}$ defined as $(g, \psi) \mapsto \pi(g) \psi)$ is continuous. The homomorphism condition is equivalent to requiring that $\pi(g)$ is a continuous linear operator on $\mathcal{H}$ for all $g \in G$, that $\pi(e)=\mathbb{I}$ ( $e$ being the identity element of the group) and that the product is preserved, i.e. $\pi\left(g_{1} g_{2}\right)=\pi\left(g_{1}\right) \pi\left(g_{2}\right)$ for all $g_{1}, g_{2} \in G$.

If $\pi^{\prime}$ is another representation of $G$ in $\mathcal{H}^{\prime}$, a continuous linear map $T: \mathcal{H} \rightarrow \mathcal{H}^{\prime}$ is called an intertwining operator if $T \circ \pi(g)=\pi^{\prime}(g) \circ T$ for all $g \in G$. This would be the "quantum" version of our "related" vector fields considered in the classical setting. If $T$ is an isomorphism, $\pi$ and $\pi^{\prime}$ are said to be isomorphic. Given a representation $\pi$, the continuous operators $T: \mathcal{H} \rightarrow \mathcal{H}$ which intertwine $\pi$ with itself, constitute an algebra, called the commuting algebra of the representation $\pi$. For unitary representations, the isomorphic representations are unitarily equivalent.

For a unitary representation $\pi: G \rightarrow U(\mathcal{H})$, we may consider the set of $C^{\infty}$ vectors (denoted as $\mathcal{H}^{\infty}$ ) and the set of analytic vectors (denoted as $\mathcal{H}^{\omega}$ ). According to Nelson 48, both of them are always dense subspaces of the Hilbert space $\mathcal{H}$. Thus it is possible to derive a representation of the Lie algebra $\mathfrak{g}$ of $G$ on these subspaces by setting

given by

$$
\dot{\pi}: \mathfrak{g} \rightarrow \operatorname{End}\left(\mathcal{H}^{\infty}\right)
$$

$$
\dot{\pi}(x) \psi=\lim _{t \rightarrow 0} \frac{1}{t}(\pi(\exp (t x)) \psi-\psi) .
$$

This representation has a natural extension to a representation of the universal enveloping algebra $U(\mathfrak{g})$, which is still denoted by $\dot{\pi}$.

If we define an involution on $U(\mathfrak{g})$ by setting

$$
X^{*}=-X \quad \forall X \in \mathfrak{g},
$$

the extension $\dot{\pi}$ to $U(\mathfrak{g})$ is a $*$-representation in the sense that for all $Y \in U(\mathfrak{g})$

$$
\dot{\pi}\left(Y^{*}\right) \subseteq(\dot{\pi}(Y))^{*} .
$$

It is possible to introduce states on the universal enveloping algebra by means of linear functionals. A functional $\Phi: U(\mathfrak{g}) \rightarrow \mathbb{C}$ is said to be a state if

$$
\Phi\left(X^{*} X\right) \geq 0 \quad \forall X \in U(\mathfrak{g}) .
$$

This implies that $\Phi\left(X^{*}\right)=\bar{\Phi}(X)$ for all the elements $X \in U(\mathfrak{g})$. Convex combinations of states are also states, and thus they form a cone which defines a partial ordering on the linear space of all self-adjoint linear functionals on $U(\mathfrak{g})$. Often, we consider normalized functionals defined as $\Phi(\mathbb{I})=1$. The states that can not be written as convex combinations are extremal states and are called pure states.

By using the Gelfand-Naimark-Segal (GNS) construction, with any state $\Phi$, we associate a Hilbert space $\mathcal{H}_{\Phi}$, and a $*$-representation $\sigma$ of $U(\mathfrak{g})$ by (unbounded) operators on a dense subspace $\mathcal{D} \subset \mathcal{H}_{\Phi}$. There exists also a vector $\Omega \in \mathcal{D}$ such that for all $X \in U(\mathfrak{g})$,

$$
\langle\sigma(x) \Omega \mid \Omega\rangle=\Phi(x),
$$


and $\mathcal{D}=\sigma(\{U(\mathfrak{g})\}) . \Omega$.

If $\Phi$ is an analytic state, the representation is an irreducible unitary representation of the algebra if and only if $\Phi$ is a pure state. From the construction, it is clear that by acting with the group $G$ on $\Omega$, we identify a submanifold of states in $\mathcal{H}_{\Phi}$ which may be pulled back to $G$ and therefore put into correspondence with a Hilbert space contained in $\mathcal{L}^{2}(G)$ (the Hilbert space of square integrable functions on the group with respect to the Haar measure). Thus, in conclusion, in this approach a "classical manifold" appears again to be "built in" from the beginning via the smooth manifold represented by the Lie group $G$. Let us mention that the procedure considered in 21] shows very clearly that by using representations of $G$ on spaces which carry alternative linear structures would give rise to "intertwining maps" which are no more linear. In this way the "vector field" point of view would not be "optional" any more but would be mandatory. Moreover this would be a way to implement nonlinear transformations carrying from a Hilbert space description to another Hilbert space description not linearly related among them. A situation like this should be contemplated if we would like to have "alternative Poisson structures" non necessarily compatible among them.

We shall mention now few examples where the previous constructions can be traced in the specific realizations of groups and algebras.

3.3. Finite level systems and alternative descriptions. Within the geometrical formulation of Quantum Mechanics, a quantum system is described by a vector field $\Gamma$ which preserves a Hermitian structure. The carrier space, when considered not as a complex vector space but as a real manifold, gives rise to a Riemannian structure, a symplectic structure and a connecting complex structure (these aspects will be further elaborated in Section 4). A vector field which describes quantum evolution has to preserve all of them: this means that it is not only symplectic, it is also a Killing vector field and generates a complex linear transformation. As the dynamics is linear and the structure above is translationally invariant, we can represent the previous compatibility conditions in terms of their representative matrices.

We have

Lemma 2. A matrix $A$ is the matrix associated with some linear Hamiltonian vector field if and only if it can be decomposed into the product of a skew symmetric matrix $\Lambda$ and a symmetric matrix $H$, i.e.

$$
A=\Lambda . H .
$$

Out of this decomposition we construct a Poisson bracket from $\Lambda$ and the Hamiltonian function from $H$.

If we consider now any linear invertible transformation $T: \mathcal{H} \rightarrow \mathcal{H}$, we know the transformation law of the matrix $A$ representing a linear transformation $(A \mapsto$ $T A T^{-1}$ ) and we can write, by considering $\Lambda$ and $H$ as representative of bilinear maps on account of the identification we are going to make

$$
A \mapsto A_{T}=T A T^{-1}=T \Lambda T^{t}\left(T^{t}\right)^{-1} H T^{-1}=\Lambda_{T} H_{T} .
$$

We have considered $\Lambda$ to transform contravariantly and $H$ to transform covariantly.

If $T$ is a symmetry transformation for $A$, i.e $A_{T}=A$, we conclude

$$
A=\Lambda_{T} H_{T},
$$


where, in general, $\Lambda_{T}$ will be different from $\Lambda$. If $\Lambda$ represents a Poisson structure, we would say that the transformation $T$ is a symmetry for the dynamics which is not "canonical". Thus the symmetry group for $A$, quotiented by the subgroup of canonical transformations for $\Lambda$ would parametrize the family of alternative decompositions for $A$ and consequently of the family of alternative Hamiltonian descriptions [47.

There are a few properties of these alternative descriptions which are immediate to list:

- all odd powers of $A$ are traceless, i.e.

$$
\operatorname{Tr} A^{2 k+1}=0,
$$

and give rise to new Hamiltonian systems.

- when $A$ is generic, the commutant and the bicommutant coincide and are generated by all powers of $A$.

- in the generic case

$$
e^{\lambda A^{2}} \Lambda e^{-\lambda\left(A^{T}\right)^{2}}=\Lambda_{\lambda}
$$

for different values of $\lambda$ gives representative matrices of alternative Poisson structures.

- in the quantum case, if $T$ in addition commutes with the complex structure (i.e. it is the real form of a complex linear transformation), then it will also generate alternative Hermitian structures when $H$ is positive definite. In particular, in the generic case, $e^{\lambda A^{2}}$ will generate alternative Hermitian structures (if we write $A=i B$ where $B$ is Hermitian, it is evident that the even powers of $A$ are not skew-Hermitian).

By using collective coordinates, say $\left\{\xi_{j}\right\}$, for the realification of the complex vector space, we have for the corresponding tensor fields:

$$
\Gamma=\xi_{k} A_{j}^{k} \frac{\partial}{\partial \xi_{j}} ; \quad \Lambda_{\lambda}=\left(\Lambda_{\lambda}\right)_{j k} \frac{\partial}{\partial \xi_{j}} \wedge \frac{\partial}{\partial \xi_{k}} ; \quad H_{\lambda}=\frac{1}{2} H_{\lambda}^{j k} \xi_{j} \xi_{k},
$$

which explain the transformation properties we have used for $\Lambda$ and $H$. We obtain thus alternative Hamiltonian descriptions. It is important to remark that the alternative Poisson structures are mutually compatible, i.e. any combination of them with real valued coefficients is still a Poisson structure.

To be more definite, we shall consider a specific example given by a quantum system with a 2-dimensional Hilbert space (i.e. a two-level quantum system). The equations of the motion for such a system would be:

$$
\dot{S}=[H, S] \quad S=S_{1} \sigma_{1}+S_{2} \sigma_{2}+S_{3} \sigma_{3} \quad H=B \sigma_{3}
$$

The matrix $\sigma_{3}$ may be considered to be the representative matrix of our dynamics, thus

$$
A=i\left(\begin{array}{cc}
1 & 0 \\
0 & -1
\end{array}\right) \quad A^{2}=\left(\begin{array}{cc}
-1 & 0 \\
0 & -1
\end{array}\right) \quad A^{4}=\left(\begin{array}{ll}
1 & 0 \\
0 & 1
\end{array}\right)
$$

This case is not generic, and therefore we have to consider matrices in the commutant of $\sigma_{3}$ which are skew Hermitian. Without going through the full procedure, it is not difficult to see that all alternative Hamiltonian descriptions may be written in the form

$$
h=a_{1}^{2} z_{1} \bar{z}_{1}+a_{2}^{2} z_{2} \bar{z}_{2} \quad a_{1}, a_{2} \in \mathbb{R}
$$


and in real coordinates $\left(z_{j}=q_{j}+i p_{j}\right)$ we would have

$$
h=a_{1}^{2}\left(p_{1}^{2}+q_{1}^{2}\right)+a_{2}^{2}\left(p_{2}^{2}+q_{2}^{2}\right) \quad a_{1}, a_{2} \in \mathbb{R} ;
$$

along with corresponding symplectic and Riemannian structures

$$
g=a_{1}^{2}\left(d p_{1}^{2}+d q_{1}^{2}\right)+a_{2}^{2}\left(d p_{2}^{2}+d q_{2}^{2}\right) \quad \omega=a_{1}^{2} d p_{1} \wedge d q_{1}+a_{2}^{2} d p_{2} \wedge d q_{2}
$$

A further generalization arise if we let $a_{1}$ to become a function of $z_{1} \bar{z}_{1}$ and $a_{2}$ a function of $z_{2} \bar{z}_{2}$ (see [18]), or more generally

$$
h=a_{1}^{2}\left(z_{1} \bar{z}_{1}, z_{2} \bar{z}_{2}\right) z_{1} \bar{z}_{1}+z_{2} \bar{z}_{2} a_{2}^{2}\left(z_{1} \bar{z}_{1}, z_{2} \bar{z}_{2}\right) .
$$

In this case the skew-symmetric part of the Hermitian tensor is still given by a symplectic structure, but the Riemannian part is no longer flat. If we use a Darboux chart for the new symplectic structure, we can define a new flat Riemannian tensor, and hence a new Hermitian structure, but it will not be connected to the original one by nonlinear "point transformations", i.e. transformations only on the "coordinates". To put it differently, the Darboux coordinates are adapted to the symplectic structure but are "not adapted" to the Riemannian tensor. The new Darboux chart allows to introduce a new linear structure such that the original dynamics is still linear with respect to the new linear structure. On each symplectic plane the metric tensor is going to be

$$
\left(d \rho_{1}\right)^{2}+\rho_{1}^{2}\left(d \phi_{1}\right)^{2}
$$

in the flat case, and there will be a conformal factor instead when $a_{j}$ is a function of the coordinates.

By using the new linear structure associated with the Darboux chart, it is possible to define a new realization of the unitary group $U(2)$ which is not linearly related to the original one 21].

To be able to make sense of some of these nonlinear manipulations, it is convenient to consider a geometrical formulation of Quantum Mechanics.

\section{Geometric Quantum Mechanics}

The aim of this section is to present a brief summary of the set of the geometrical tools which characterize the description of Quantum Mechanics. For further details we refer the reader to $26,27,7,8,13,14$.

4.1. The states. To introduce the real manifold point of view, we start by replacing the Hilbert space $\mathcal{H}$ with its realification $\mathcal{H}_{\mathbb{R}}$. In this realification process the complex structure on $\mathcal{H}$ will be represented by a tensor $J$ on $\mathcal{H}_{\mathbb{R}}$ as we will see.

The natural identification is then provided by

$$
\psi_{R}+i \psi_{I}=\psi \in \mathcal{H} \mapsto\left(\psi_{R}, \psi_{I}\right) \in \mathcal{H}_{\mathbb{R}} .
$$

Under this transformation, the Hermitian product becomes, for $\psi^{1}, \psi^{2} \in \mathcal{H}$

$$
\left\langle\left(\psi_{R}^{1}, \psi_{I}^{1}\right),\left(\psi_{R}^{2}, \psi_{I}^{2}\right)\right\rangle=\left(\left\langle\psi_{R}^{1}, \psi_{R}^{2}\right\rangle+\left\langle\psi_{I}^{1}, \psi_{I}^{2}\right\rangle\right)+i\left(\left\langle\psi_{R}^{1}, \psi_{I}^{2}\right\rangle-\left\langle\psi_{I}^{1}, \psi_{R}^{2}\right\rangle\right) .
$$

To consider $\mathcal{H}_{\mathbb{R}}$ just as a real differential manifold, the algebraic structures available on $\mathcal{H}$ must be converted into tensor fields on $\mathcal{H}_{\mathbb{R}}$. To this end we introduce the tangent bundle $T \mathcal{H}_{\mathbb{R}}$ and its dual the cotangent bundle $T^{*} \mathcal{H}_{\mathbb{R}}$. The linear structure available in $\mathcal{H}_{\mathbb{R}}$ is encoded in the vector field $\Delta$

$$
\Delta: \mathcal{H}_{\mathbb{R}} \rightarrow T \mathcal{H}_{\mathbb{R}} \quad \psi \mapsto(\psi, \psi)
$$


We can consider the Hermitian structure on $\mathcal{H}_{R}$ as an Hermitian tensor on $T \mathcal{H}_{\mathbb{R}}$. With every vector we can associate a vector field

$$
X_{\psi}: \mathcal{H}_{\mathbb{R}} \rightarrow T \mathcal{H}_{\mathbb{R}} \quad \phi \rightarrow(\phi, \psi)
$$

These vector fields are the infinitesimal generators of the vector group $\mathcal{H}_{\mathbb{R}}$ acting on itself.

Therefore, the Hermitian tensor, denoted in the same way as the scalar product will be

$$
\left\langle X_{\psi_{1}}, X_{\psi_{2}}\right\rangle(\phi)=\left\langle\psi_{1}, \psi_{2}\right\rangle
$$

i.e. the tensor field we are defining is translationally invariant.

On the "real manifold" the Hermitian scalar product may be written as $\left\langle\psi_{1}, \psi_{2}\right\rangle=$ $g\left(X_{\psi_{1}}, X_{\psi_{2}}\right)+i \omega\left(X_{\psi_{1}}, X_{\psi_{2}}\right)$, where $g$ is now a symmetric tensor and $\omega$ a skewsymmetric one. It is also possible to write the associated quadratic form as a pull-back by means of the dilation vector field $\Delta$ extended as a map in an obvious way $\mathcal{H}_{R} \rightarrow T \mathcal{H}_{\mathbb{R}} \oplus T \mathcal{H}_{\mathbb{R}}$ :

$$
\left(\Delta^{*}(g+i \omega)\right)(\psi, \psi)=\langle\psi, \psi\rangle_{\mathcal{H}}
$$

The properties of the Hermitian product ensure that:

- the symmetric tensor is positive definite and non-degenerate, and hence defines a Riemannian structure on the real vector manifold.

- the skew-symmetric tensor is also non degenerate, and is closed with respect to the natural differential structure of the vector space. Hence, the tensor is a symplectic form.

As the inner product is sesquilinear, it satisfies

$$
\left\langle\psi_{1}, i \psi_{2}\right\rangle=i\left\langle\psi_{1}, \psi_{2}\right\rangle, \quad\left\langle i \psi_{1}, \psi_{2}\right\rangle=-i\left\langle\psi_{1}, \psi_{2}\right\rangle .
$$

This implies

$$
g\left(X_{\psi_{1}}, X_{\psi_{2}}\right)=\omega\left(J X_{\psi_{1}}, X_{\psi_{2}}\right)
$$

We also have that $J^{2}=-\mathbb{I}$, and hence that the triple $(J, g, \omega)$ defines a Kähler structure. This implies, among other things, that the tensor $J$ generates both finite and infinitesimal transformations which are orthogonal and symplectic.

Linear transformations on the vector space $\mathcal{H}_{\mathbb{R}}$ are converted into $(1,1)$-tensor fields by setting $A \rightarrow T_{A}$ where

$$
T_{A}: T \mathcal{H}_{\mathbb{R}} \rightarrow T \mathcal{H}_{\mathbb{R}} \quad(\psi, \phi) \mapsto(\psi, A \phi) .
$$

The association $A \rightarrow T_{A}$ is an associative algebra isomorphism. The Lie algebra of vector fields obtains by setting $X_{A}=T_{A}(\Delta)$. Complex linear transformations will be represented by $(1,1)$-tensor fields commuting with $J$, or by vector fields $Y$ such that $L_{Y} J=0$.

For finite dimensional Hilbert spaces it may be convenient to introduce adapted coordinates on $\mathcal{H}$ and $\mathcal{H}_{\mathbb{R}}$. An orthonormal basis $\left\{\left|e_{k}\right\rangle\right\}$ of the Hilbert space allows us to identify this product with the canonical Hermitian product on $\mathbb{C}^{n}$ :

$$
\left\langle\psi_{1}, \psi_{2}\right\rangle=\sum_{k}\left\langle\psi_{1}, e_{k}\right\rangle\left\langle e_{k}, \psi_{2}\right\rangle
$$

The group of unitary transformations on $\mathcal{H}$ becomes identified with the group $U(n, \mathbb{C})$, its Lie algebra $\mathfrak{u}(\mathcal{H})$ with $\mathfrak{u}(n, \mathbb{C})$ and so on. 
The choice of the basis also allows us to introduce coordinates for the realified structure:

$$
\left\langle e_{k}, \psi\right\rangle=\left(q_{k}+i p_{k}\right)(\psi),
$$

and write the geometrical structures introduced above as:

$$
J=\partial_{p_{k}} \otimes d q_{k}-\partial_{q_{k}} \otimes d p_{k} \quad g=d q_{k} \otimes d q_{k}+d p_{k} \otimes d p_{k} \quad \omega=d q_{k} \wedge d p_{k}
$$

If we combine them into complex coordinates we can write the Hermitian structure by means of $z_{n}=q_{n}+i p_{n}$ :

$$
h=\sum_{k} d \bar{z}_{k} \otimes d z_{k}
$$

In an analogous way we can consider a contravariant version of these tensors. It is also possible to build it by using the isomorphism $T \mathcal{H}_{\mathbb{R}} \leftrightarrow T^{*} \mathcal{H}_{\mathbb{R}}$ associated to the Riemannian tensor $g$. The result in both cases is a Kähler structure for the dual vector space $\mathcal{H}_{\mathbb{R}}^{*}$ with the dual complex structure $J^{*}$, a Riemannian tensor $G$ and a (symplectic) Poisson tensor $\Omega$ : The coordinate expressions with respect to the natural basis are:

- the Riemannian structure $G=\sum_{k=1}^{n}\left(\frac{\partial}{\partial q_{k}} \otimes \frac{\partial}{\partial q_{k}}+\frac{\partial}{\partial p_{k}} \otimes \frac{\partial}{\partial p_{k}}\right)$,

- the Poisson tensor $\Omega=\sum_{k=1}^{n}\left(\frac{\partial}{\partial q_{k}} \wedge \frac{\partial}{\partial p_{k}}\right)$

- while the complex structure has the form

$$
J=\sum_{k=1}^{n}\left(\frac{\partial}{\partial p_{k}} \otimes d q_{k}-\frac{\partial}{\partial q_{k}} \otimes d p_{k}\right)
$$

4.2. The observables. The space of observables (i.e. of self-adjoint operators acting on $\mathcal{H})$ may be identified with the dual $\mathfrak{u}^{*}(\mathcal{H})$ of the real Lie algebra $\mathfrak{u}(\mathcal{H})$, according to the pairing between the unitary Lie algebra and its dual given by

$$
A(T)=\frac{i}{2} \operatorname{Tr} A T
$$

Under the previous isomorphism, $\mathfrak{u}^{*}(\mathcal{H})$ becomes a Lie algebra with product defined by

$$
[A, B]=\frac{\hbar}{2 i}(A B-B A)
$$

We can also consider the Jordan product:

$$
[A, B]_{+}=2(A \circ B)=A B+B A
$$

with the associative product given by

$$
A B=A \circ B+\frac{i}{\hbar}[A, B]
$$

and defined on the complexified space $\mathfrak{g l}(\mathcal{H})$.

Both structures are compatible in the sense that the Jordan product is invariant under the linear transformations on $\mathfrak{u}^{*}(\mathcal{H})$ defined by the adjoint action (of the Poisson product). As a result, $\mathfrak{u}^{*}(\mathcal{H})$ becomes a Lie-Jordan algebra (see [19, 32]).

We can also define a suitable scalar product on Hermitian operators, given by:

$$
\langle A, B\rangle=\frac{1}{2} \operatorname{Tr} A B
$$

which turns the real vector space into a real Hilbert space. This scalar product is the restriction of the one on $\mathfrak{g l}(\mathcal{H})$ defined as $\langle M, N\rangle=\frac{1}{2} \operatorname{Tr} M^{\dagger} N$. 
Moreover this scalar product is compatible with the Lie-Jordan structure in the following sense:

$$
\langle[A, \xi], B\rangle_{\mathfrak{u}^{*}(\mathcal{H})}=\langle A,[\xi, B]\rangle_{\mathfrak{u}^{*}(\mathcal{H})} \quad\left\langle[A, \xi]_{+}, B\right\rangle_{\mathfrak{u}^{*}(\mathcal{H})}=\left\langle A,[\xi, B]_{+}\right\rangle_{\mathfrak{u}^{*}(\mathcal{H})}
$$

These algebraic structures may be given a tensorial formulation in terms of the association $A \mapsto T_{A}$ on the tangent bundle $T \mathfrak{u}^{*}(\mathcal{H})$. However we can also associate complex valued functions with linear operators $A \in \mathfrak{g l}(\mathcal{H})$ by means of the scalar product

$$
\mathfrak{g l}(\mathcal{H}) \ni A \mapsto f_{A}=\frac{1}{2}\langle\psi, A \psi\rangle_{\mathcal{H}}
$$

In more intrinsic terms we may write

$$
f_{A}=\frac{1}{2}\left(g\left(\Delta, X_{A}\right)+i \omega\left(\Delta, X_{A}\right)\right) .
$$

Hermitian operators give rise thus to quadratic real valued functions.

The association of operators with quadratic functions allows also to recover the product structures on $\mathfrak{u}(\mathcal{H})$ and $\mathfrak{u}^{*}(\mathcal{H})$ by means of appropriate $(0,2)$-tensors on $\mathcal{H}_{\mathbb{R}}$. Indeed, by using the contravariant form of the Hermitian tensor $G+i \Omega$ given by:

$$
G+i \Omega=4 \frac{\partial}{\partial z_{k}} \otimes \frac{\partial}{\partial \bar{z}_{k}}=\frac{\partial}{\partial q_{k}} \otimes \frac{\partial}{\partial q_{k}}+\frac{\partial}{\partial p_{k}} \otimes \frac{\partial}{\partial p_{k}}+i \frac{\partial}{\partial q_{k}} \wedge \frac{\partial}{\partial p_{k}},
$$

it is possible to define a bracket

$$
\{f, h\}_{\mathcal{H}}=\{f, h\}_{g}+i\{f, h\}_{\omega}
$$

We remark that $\frac{\partial}{\partial z_{k}}$ and $\frac{\partial}{\partial \bar{z}_{k}}$ are not to be considered as derivations with respect to the complex coordinates introduced above but as complex valued smooth vector fields.

In particular, for quadratic real valued functions we have

$$
\left\{f_{A}, f_{B}\right\}_{g}=f_{A B+B A}=2 f_{A \circ B} \quad\left\{f_{A}, f_{B}\right\}_{\omega}=-i f_{A B-B A}
$$

The imaginary part, i.e. $\{\cdot, \cdot\}_{\omega}$, defines a Poisson bracket on the space of functions. Both brackets define a tensorial version of the Lie-Jordan algebra for the set of operators.

For Hermitian operators we recover previously defined vector fields:

$$
\operatorname{grad} f_{A}=\widetilde{A} ; \quad \operatorname{Ham} f_{A}=\widetilde{i A}
$$

where the vector fields associated with operators, we recall, are defined by:

$$
\begin{gathered}
\widetilde{A}: \mathcal{H}_{\mathbb{R}} \rightarrow T \mathcal{H}_{R} \quad \psi \mapsto(\psi, A \psi) \\
\widetilde{i A}: \mathcal{H}_{\mathbb{R}} \rightarrow T \mathcal{H}_{R} \quad \psi \mapsto(\psi, J A \psi)
\end{gathered}
$$

We can also consider the algebraic structure associated to the full bracket $\{\cdot, \cdot\}_{\mathcal{H}}$, as we associated above the Jordan product and the commutator of operators to the brackets $\{\cdot, \cdot\}_{g}$ and $\{\cdot, \cdot\}_{\omega}$ respectively. It is simple to see that it corresponds to the associative product of the set of operators, i.e.

$$
\left\{f_{A}, f_{B}\right\}_{\mathcal{H}}=\left\{f_{A}, f_{B}\right\}_{g}+i\left\{f_{A}, f_{B}\right\}_{\omega}=f_{A B+B A}+f_{A B-B A}=2 f_{A B}
$$

This particular bilinear product on quadratic functions may be used to define a star product on quadratic functions

$$
\left\{f_{A}, f_{B}\right\}_{\mathcal{H}}=2 f_{A B}=\left\langle d f_{A}, d f_{B}\right\rangle_{\mathcal{H}^{*}}=f_{A} \star f_{B}
$$


The set of quadratic functions endowed with such a product turns out to be a $\mathbb{C}^{*}$-algebra.

Summarizing, we can reconstruct all the information of the algebra of operators starting only with real-valued functions defined on $\mathcal{H}_{\mathbb{R}}$. We have thus

Proposition 1 ([12]). The Hamiltonian vector field $X_{f}$ (defined as $X_{f}=\hat{\Omega}(d f)$ ) is a Killing vector field for the Riemannian tensor $G$ if and only if $f$ is a quadratic function associated with an Hermitian operator $A$, i.e. there exists $A=A^{\dagger}$ such that $f=f_{A}$.

Finally, we can consider the problem of how to recover the eigenvalues and eigenvectors of the operators at the level of the functions of $\mathcal{H}_{R}$. We consider the expectation value functions associated to the operators as:

$$
A \mapsto e_{A}(\psi)=\frac{\langle\psi, A \psi\rangle}{\langle\psi, \psi\rangle} .
$$

Then,

- eigenvectors correspond to the critical points of functions $e_{A}$, i.e.

$$
d e_{A}\left(\psi_{a}\right)=0
$$

iff $\psi_{a}$ is an eigenvector of $A$. We notice that the invariance of $e_{A}$ under multiplication by a phase $U(1)$ implies that critical points form a circle on the sphere of normalized vectors if the eigenvalue is not degenerate.

- the corresponding eigenvalue is recovered by the value $e_{A}\left(\psi_{a}\right)$

Thus we can conclude that the Kähler manifold $\left(\mathcal{H}_{\mathbb{R}}, J, \omega, g\right)$ contains all the information of the usual formulation of Quantum Mechanics on a complex Hilbert space.

Up to now we have concentrated our attention on states and observables. If we consider observables as generators of transformations, i.e. we consider the Hamiltonian flows associated to the corresponding functions, the invariance of the tensor $G$ implies that the evolution is actually unitary. It is, therefore, natural, to consider the action of the unitary group on the realification of the complex vector space.

4.3. The momentum map: geometrical structures on $\mathfrak{u}^{*}$. The unitary action of $U(\mathcal{H})$ on $\mathcal{H}$ induces a symplectic action on the symplectic manifold $\left(\mathcal{H}_{\mathbb{R}}, \omega\right)$. By using the association

$$
F: \mathcal{H}_{\mathbb{R}} \times \mathfrak{u}(\mathcal{H}) \rightarrow \mathbb{R} \quad(\psi, A) \mapsto \frac{1}{2}\langle\psi, i A \psi\rangle=f_{i A}(\psi),
$$

we find, with $F_{A}=f_{i A}: \mathcal{H}_{\mathbb{R}} \rightarrow \mathbb{R}$, that

$$
\{F(A), F(B)\}_{\omega}=i F([A, B]) .
$$

Thus if we fix $\psi$, we have a mapping $F(\psi): \mathfrak{u}(\mathcal{H}) \rightarrow \mathbb{R}$. With any element $\psi \in \mathcal{H}$ we associate an element in $\mathfrak{u}^{*}(\mathcal{H})$. The previous map defines a momentum map

$$
\mu: \mathcal{H} \rightarrow \mathfrak{u}^{*}(\mathcal{H})
$$

which provides us with a symplectic realization of the natural Poisson manifold structure available in $\mathfrak{u}^{*}(\mathcal{H})$. We can write the momentum map from $\mathcal{H}_{\mathbb{R}}$ to $\mathfrak{u}^{*}(\mathcal{H})$ as

$$
\mu(\psi)=|\psi\rangle\langle\psi|
$$


If we make the convention that the dual $\mathfrak{u}^{*}(\mathcal{H})$ of the (real) Lie algebra $\mathfrak{u}(\mathcal{H})$ is identified with Hermitian operators by means of a scalar product, the product pairing between Hermitian operators $A \in \mathfrak{u}^{*}(\mathcal{H})$ and the anti-Hermitian element $T \in \mathfrak{u}(\mathcal{H})$ will be given by

$$
A(T)=\frac{i}{2} \operatorname{Tr}(A T)
$$

If we denote the linear function on $\mathfrak{u}^{*}(\mathcal{H})$ associated with the element $i A \in \mathfrak{u}(\mathcal{H})$ by $\hat{A}$, we have

$$
\mu^{*}(\hat{A})=f_{A}
$$

The pullback of linear functions on $u^{*}(\mathcal{H})$ is given by the quadratic functions on $\mathcal{H}_{\mathbb{R}}$ associated with the corresponding Hermitian operators.

It is possible to show that the contravariant tensor fields on $\mathcal{H}_{\mathbb{R}}$ associated with the Hermitian structure are $\mu$-related with a complex tensor on $\mathfrak{u}^{*}(\mathcal{H})$ :

$$
\mu_{*}(G+i \Omega)=R+i \Lambda
$$

where the two new tensors $R$ and $\Lambda$ are defined by

$$
[R(\hat{A}, \hat{B})](\xi)=\left\langle\xi,[A, B]_{+}\right\rangle_{\mathfrak{u}^{*}}=\frac{1}{2} \operatorname{Tr}(\xi(A B+B A))
$$

and

Clearly,

$$
[\Lambda(\hat{A}, \hat{B})](\xi)=\left\langle\xi,[A, B]_{-}\right\rangle_{\mathfrak{u}^{*}}=\frac{1}{2 i} \operatorname{Tr}(\xi(A B-B A))
$$

$$
G\left(\mu^{*} \hat{A}, \mu^{*} \hat{B}\right)+i \Omega\left(\mu^{*} \hat{A}, \mu^{*} \hat{B}\right)=\mu^{*}(R(\hat{A}, \hat{B})+i \Lambda(\hat{A}, \hat{B})) .
$$

4.4. Example. Let us consider the manifold $\mathbb{R}^{2 n}$ and the dynamical system

$$
\Gamma=p_{j} \frac{\partial}{\partial q_{j}}-\nu^{2} q_{j} \frac{\partial}{\partial p_{j}} .
$$

We can find alternative Hamiltonian structures connected to the factorization of

$$
\Gamma=\left(q_{j}, p_{j}\right)\left(\begin{array}{cc}
0 & -\nu^{2} \\
1 & 0
\end{array}\right)\left(\begin{array}{c}
\frac{\partial}{\partial q_{j}} \\
\frac{\partial}{\partial p_{j}}
\end{array}\right)=p_{j} \frac{\partial}{\partial q_{j}}-\nu^{2} q_{j} \frac{\partial}{\partial p_{j}}
$$

where

$$
\left(\begin{array}{cc}
0 & -\nu^{2} \\
1 & 0
\end{array}\right)=\left\{\begin{array}{c}
\left(\begin{array}{cc}
0 & -1 \\
1 & 0
\end{array}\right)\left(\begin{array}{cc}
1 & 0 \\
0 & \nu^{2}
\end{array}\right) \\
\left(\begin{array}{cc}
0 & -\nu^{2} \\
\nu^{2} & 0
\end{array}\right)\left(\begin{array}{cc}
\frac{1}{\nu^{2}} & 0 \\
0 & 1
\end{array}\right)
\end{array}\right.
$$

In the case of $n=2$, by redefining position and momenta as $(q, p) \rightarrow\left(q^{\prime}, p\right)$, according to the symmetric part of the decomposition we have:

$$
\Gamma=\nu\left(p_{j} \frac{\partial}{\partial q_{j}^{\prime}}-q_{j}^{\prime} \frac{\partial}{\partial p_{j}}\right)
$$

Let us now consider in $\mathbb{R}^{4}$ the Hamiltonian function

$$
H=\frac{1}{2} \nu\left(p_{1}^{2}+q_{1}^{2}+p_{2}^{2}+q_{2}^{2}\right),
$$

and the corresponding flow.

Therefore, $H=\frac{1}{2} \nu$ implies $p_{1}^{2}+q_{1}^{2}+p_{2}^{2}+q_{2}^{2}=1$, i.e. a point in the unit sphere. A family of constants of the motion is provided by the set

$$
p_{1} q_{2}-q_{1} p_{2} \quad p_{1} p_{2}+q_{1} q_{2} \quad\left(p_{1}^{2}+q_{1}^{2}\right)-\left(p_{2}^{2}+q_{2}^{2}\right) .
$$


It is simple to check that their Poisson bracket generate the Lie algebra of $S U(2)$ and along with the dynamics they generate the Lie algebra of the full unitary group $U(2)$. A foliation by two dimensional tori is given by the level sets of

$$
p_{1}^{2}+q_{1}^{2}, \quad p_{2}^{2}+q_{2}^{2},
$$

on the open dense submanifold where they are independent.

We notice that alternative Hamiltonian descriptions for $\Gamma$ may be related to the immersion of $\Gamma$ into different Lie groups or Lie algebras. For instance, we have seen that we may generate $U(2)$ when we use the Poisson bracket defined as

$$
\left\{q_{a}, p_{b}\right\}=\delta_{a b} \quad\left\{q_{a}, q_{b}\right\}=0=\left\{p_{a}, p_{b}\right\} .
$$

But if instead we consider

$$
\left\{q_{1}, p_{1}\right\}=1, \quad\left\{q_{2}, p_{2}\right\}=-1, \quad\left\{q_{a}, q_{b}\right\}=0=\left\{p_{a}, p_{b}\right\} ;
$$

the same constants of the motion will generate the Lie algebra of the group $S U(1,1)$. This would correspond to the case of the factorization of the matrix $A$ associated with the linear dynamics into a skew-symmetric and a symmetric one which is not degenerate but it is not positive definite.

It is also possible to consider the dynamics as part of an infinite dimensional Lie algebra (deformations).

In general we look for factorizations of the vector field by means of a Poisson tensor and a differential one form $(\Gamma=\Lambda(d H))$, in this way both $\Lambda$ and $d H$ need not be associated with matrices. In particular if we consider the $(1,1)$-tensor field

$$
T=\left(d p_{j} \otimes \frac{\partial}{\partial q_{j}}-d q_{j} \otimes \frac{\partial}{\partial p_{j}}\right),
$$

with any constant of the motion of the form

$$
F=F\left(p_{1}^{2}+q_{1}^{2}, p_{2}^{2}+q_{2}^{2}, p_{1} q_{2}-q_{1} p_{2}, p_{1} p_{2}+q_{1} q_{2}\right),
$$

we can associate an invariant one form 47

$$
\theta_{F}=d_{T} F \text {. }
$$

By a proper choice of the function $F$, we can make the two form $d d_{T} F$ non degenerate, i.e. we can choose $F$ in such a way that the two form

$$
\omega_{F}=d d_{T} F,
$$

is an invariant symplectic two form.

Moreover

$$
L_{\Gamma} d_{T} F=i_{\Gamma} d d_{T} F+d i_{\Gamma} d_{T} F=0
$$

implies that

$$
i_{\Gamma} d d_{T} F=-d\left(i_{\Gamma} d_{T} F\right)
$$

with

$$
i_{\Gamma} d_{T} F=d F(T(\Gamma))=-\nu d F(\Delta)=-\nu L_{\Delta} F
$$

because $T(\Gamma)=-\nu \Delta, \Delta$ being the dilation vector field.

Any other invariant $(1,1)$-tensor field will give rise to alternative symplectic structure with the same procedure.

More generally, if $X_{j}$ and $X_{j+n}$ are pairwise commuting vector fields $\left[X_{j}, X_{k}\right]=0$ $\forall j, k=1, \cdots, 2 n$, with

$$
L_{\Gamma} X_{j}=X_{j+n} \quad L_{\Gamma} X_{j+n}=-X_{j} ;
$$


the one form

$$
\theta_{F}=\frac{1}{2} \sum_{j} L_{X_{j}} d\left(L_{X_{j+n}} F\right)-L_{X_{j+n}} d\left(L_{X_{j}} F\right)
$$

is $\Gamma$-invariant and under proper choices of $F$ provides a symplectic structure

$$
\omega_{F}=d \theta_{F} .
$$

In addition, if we set

$$
Q_{j}=L_{X_{j}} F \quad P_{j}=L_{X_{j+n}} F
$$

we find a new linear structure and a new Kähler structure invariant under $\Gamma$ :

$$
L_{\Gamma} Q_{j}=P_{j} \quad L_{\Gamma} P_{j}=-Q_{j} .
$$

The new Hamiltonian function is given by

$$
\frac{1}{2}\left(\left(L_{X_{j}} F\right)^{2}+\left(L_{X_{j+n}} F\right)^{2}\right)=\frac{1}{2} \sum_{j}\left(Q_{j}^{2}+P_{j}^{2}\right),
$$

for any constant of the motion $F$, which makes $\omega_{F}$ not degenerate.

This construction of alternative Kählerian structures applies therefore to the case of quantum systems along the lines of [21].

By using the Lie algebra generated by

$$
p_{j}, q_{j}, \mathbb{I},
$$

we can consider a larger algebra by using an infinitesimal generator of an element in the automorphism group of the algebra itself

$$
\frac{1}{2} \sum_{j}\left(p_{j}^{2}+q_{j}^{2}\right) .
$$

In general, any quadratic Hamiltonian may be considered as an element of the automorphism group, the inhomogeneous symplectic group. In particular we may find subalgebras or subgroups which contain just one quadratic Hamiltonian and the full linear part. This would be a generalization of what is known as the oscillator group, which is four dimensional [52].

It is interesting to notice that the oscillator group allows for a "contraction" by letting $\nu \rightarrow 0$ and therefore it can reproduce the free particle case. On the other hand, considering the unfolding procedure described in [4, 5, it is also possible to describe the Hydrogen atom (Kepler problem) by using a nonlinear Harmonic oscillator (i.e. by introducing an energy-dependent period to be able to accommodate for the energy-period theorem when dealing with the central-force problem).

\section{The Notion of indePendence}

5.1. Proposal based on the geometric framework. Let us go back again to the problem discussed above originated by the von Neumann theorem, but from a more constructive perspective. Let us see how the tools we introduced within the geometric description of quantum mechanics allow us to introduce a notion of independence which may be convenient for our purposes.

Let us consider an even dimension vector space $V$ and a linear differential equation associated to an operator $A$ :

$$
\dot{x}=A x \quad x \in V
$$

$A$ is defining a vector field on $V$. We can consider: 
- there is a symplectic form $\omega$ on $V$ such that the vector field is Hamiltonian.

- Solve the inverse problem of Hamiltonian mechanics to find invariant symplectic forms

If $V$ is finite dimensional, we can obtain metric tensors (by using the Hessian of the Hamiltonian function) such that the vector field is a Killing vector field for $g$. By proper rescaling the metric tensor defined in this way, we may combine $\omega$ and the rescaled $g$ to define an Hermitian product on $V$ which is preserved by the Hamiltonian vector field. This allows us to introduce a set of quadratic functions on $V$ :

$$
B \rightarrow f_{B}=\langle x, B x\rangle,
$$

associated with Hermitian matrices with respect to the newly defined Hermitian products.

The set of (quadratic) functions can be endowed with different algebraic structures:

- the pointwise product

- the Poisson bracket associated to the symplectic form

- the non-local product which translates the product of operators at the level of quadratic functions:

$$
A B \rightarrow f_{A} \star f_{B}
$$

- the skew-symmetric part of the above, which encodes the commutator algebra of the operators:

$$
f_{A} \star f_{B}-f_{B} \star f_{A}
$$

Let us now discuss how to introduce a concept of functional independence. We have two possibilities:

- to consider the independence in the sense of the pointwise product and to use the standard differential calculus. Two functions are then said to be independent iff

$$
d f_{A} \wedge d f_{B} \neq 0
$$

- to consider independence with respect to the $\star$ product: in that case a noncommutative differential calculus is required and the issue becomes much more involved (see [51, 50, 6, 45]).

In the finite dimensional case it is simple to see that the notion associated with the pointwise product is convenient and provides a reasonable framework. Besides it has the advantage of being easily extensible to the case of infinite dimensional systems, once:

- a metric tensor compatible with the symplectic form has been defined. The definition can be taken from [40], where the operator $A$ is used: once the symplectic form has been chosen the Hamiltonian quadratic function $f_{A}$ is considered. This is a quadratic function, and hence it can be used to define a metric tensor

Then, there are two possibilities:

- it is positive definite: in this case it is used directly to write the metric tensor as

$$
g=\left(\begin{array}{ll}
d q & d p
\end{array}\right) \operatorname{Hessf}_{A}\left(\begin{array}{c}
d q \\
d p
\end{array}\right)
$$


- if it is not positive definite it can be proved (42]) that the space $V$ can be decomposed into subspaces (say $V_{1}$ and $V_{2}$ ) so that the functions may be written as

$$
f_{A}=f_{A_{1}}-f_{A_{2}}
$$

where $A_{1}$ is a matrix with entries only on $V_{1}$ and $A_{2}$ with entries only on $V_{2}$. A pseudo-metric (non definite metric) is thus obtained. The corresponding Hermitian structure will be pseudo-Hermitian on the total Hilbert space, but integrability may be then discussed on each Hilbert subspace where the metric may be considered to be Hermitian.

- the metric tensor allows to define suitable functions corresponding to the powers of the operator $A$. This is not a trivial issue, since depending on the domain of $A$, the powers need not have an associated function (the corresponding quadratic expression need not be convergent). Hence the freedom in the choice of the metric may be used to force all powers of the operator $A$ to be trace-class.

5.2. An example. Let us consider an application of this construction to a particular example. Consider a finite level system defined on $\mathcal{H}$ and an operator $A$ and its square $A^{2}$. It seems obvious to consider both operators as dependent, from the point of view of the associative operator product. But let us consider their independence from the point of view of the associated quadratic functions with respect to the differential calculus associated with their commutative product. Consider therefore

$$
f_{A}=\langle\psi|A| \psi\rangle \quad f_{A^{2}}=\left\langle\psi\left|A^{2}\right| \psi\right\rangle
$$

If the operator $A$ is self-adjoint with simple eigenvalues, we can consider the basis of eigenstates, and write:

$$
f_{A}=\sum_{j} \lambda_{j} \bar{z}_{j} z_{j} \quad f_{A^{2}}=\sum_{j} \lambda_{j}^{2} \bar{z}_{j} z_{j}
$$

If we compute the differential of each function:

$$
d f_{A}=\sum_{j} \lambda_{j}\left(z_{j} d \bar{z}_{j}+\bar{z}_{j} d z_{j}\right) \quad d f_{A^{2}}=\sum_{j} \lambda_{j}^{2}\left(z_{j} d \bar{z}_{j}+\bar{z}_{j} d z_{j}\right)
$$

And these two one forms satisfy

$\left.\left.d f_{A} \wedge d f_{A^{2}}=\sum_{j k}\left(\lambda_{j} \lambda_{k}^{2}-\lambda_{j}^{2} \lambda_{k}\right) \bar{z}_{j} z_{k} d z_{j} \wedge d \bar{z}_{k}\right)=\sum_{j k} \lambda_{j} \lambda_{k}\left(\lambda_{k}-\lambda_{j}\right) \bar{z}_{j} z_{k} d z_{j} \wedge d \bar{z}_{k}\right) \neq 0$

Thus we see that, in the generic case, these two functions can be considered to be functionally independent. The condition for this, in general, will depend on the spectrum.

\section{Remark}

We try to explain in a nutshell the main ideas involved in previous constructions.

Any algebra $A$ may be considered as a vector space $V$ along with a binary product $B: V \times V \rightarrow V$. Given any linear vector space $V$, we may "geometrize" it by considering the embedding $V \hookrightarrow \operatorname{Lin}\left(V^{*}, \mathbb{R}\right) \subset \mathcal{F}\left(V^{*}\right)$, i.e. any element $u$ of $V$ is thought of as a linear function on the dual space $V^{*}$. If $\alpha \in V^{*}$, we have

$$
\hat{u}(\alpha)=\alpha(u)
$$


i.e. $u$ is mapped into $\hat{u}$. Once we identify $\hat{u}$ with a function on $V^{*}$, it makes sense to consider polynomial functions and construct more general functions by means of the Weierstrass theorem. On this space of functions we may develop a standard exterior differential calculus.

On the other hand, the binary bilinear product available on $V$, allows to define a product on $\hat{V}$, by setting

$$
(\hat{v} \star \hat{u})(\alpha)=\alpha(B(v, u)) .
$$

This product is non-local and would require a non-commutative exterior differential calculus.

To clarify our statements, let us consider the algebra of $n \times n$ matrices $M(n, \mathbb{R})$. We may consider its dual space generated by

$$
\left\{e_{j k}=\left|e_{j}\right\rangle\left\langle e_{k}\right|\right\},
$$

constructed out of an orthonormal basis in some $n$-dimensional vector space $E$. Clearly,

$$
e_{j k}(A)=\left\langle e_{k}, A e_{j}\right\rangle
$$

defines the dual pairing. We may define thus

$$
\hat{A}\left(e_{j k}\right)=e_{j k}(A),
$$

and the products

$$
(\hat{A} \cdot \hat{B})\left(e_{j k}\right)=e_{j k}(A) \cdot e_{j k}(B)
$$

and

$$
(\hat{A} \star \hat{B})\left(e_{j k}\right)=e_{j k}(A \cdot B) .
$$

The first one is the usual Hadamard (or Shur) product among matrices and is commutative and local. The exterior differential calculus associated to it is the standard one on $\mathbb{R}^{n^{2}}$.

The second product is the standard row-by-column product, and is non-local and non-commutative.

Thus, in a sense, the first product is originated only from the vector space structure of the algebra, while the second one is specific of the binary bilinear product which defines it. Our suggestion is thus to use the exterior differential calculus associated with the first (local and commutative) product to define the notion of functional independence of observables in Quantum Mechanics.

\section{EXAmples: HARMONiC OSCILlators AND THEIR DEFormations}

From our previous discussion on classical integrability, it is obvious that Harmonic oscillators of any dimension will provide examples of integrable systems, also in the quantum framework. We will use them as a reference model to which all other quantum integrable systems should be related (those having a discrete spectrum, of course).

Now we are going to review some properties of the system and we will present then two examples of quantum integrable systems which exhibit the properties we have discussed above: the deformed Harmonic oscillator and the Coulomb problem in two and three dimensions. 
6.1. The standard Harmonic oscillator. As a preliminary simple example, we consider the Harmonic oscillator. The Hamiltonian is well known:

$$
H=\frac{1}{2}\left(P^{2}+Q^{2}\right)
$$

supposing $\hbar=1=\omega$.

The dynamics is linear and can be integrated easily. This system is obviously integrable from our point of view, and in both schemes (vector fields or operator subalgebras) the mapping $\Phi$ is the identity.

To deal with the Harmonic oscillator one introduces creation and annihilation operators $a, a^{\dagger}$ and the vacuum state $|0\rangle$ and builds the states of the Fock space as

$$
|n\rangle=\frac{\left(a^{\dagger}\right)^{n}}{\sqrt{n !}}|0\rangle .
$$

These are eigenstates of the number operator $\hat{n}=a^{\dagger} a$, and thus define the eigenstates of the Hamiltonian which is written as

$$
H=\hat{n}+\frac{1}{2} \text {. }
$$

The operators satisfy the commutation relation

$$
\left[a, a^{\dagger}\right]=\mathbb{I}
$$

and the number operator $\left(n=a^{+} a\right)$ thus generating together with the identity, the well known Heisenberg-Weyl group $\left(H_{4}\right)$.

The Hilbert space structure is fixed by the usual scalar product

$$
\langle n \mid m\rangle=\delta_{n m} .
$$

We notice that in the geometric framework these two objects determine the Riemannian and Poisson structures of our description.

In the Heisenberg picture the equations of the motion are written as

$$
\dot{a}=-i a \quad \dot{a}^{\dagger}=i a^{\dagger}
$$

It is well known that the number operator classifies the irreducible representations, as the Casimir corresponding to the subgroup $U(1) \subset H_{4}$. The corresponding Hilbert space is the well known Fock space $V$.

Our aim is to show how the same dynamics can be described on the space of states endowed with different Hermitian structures (therefore on different Hilbert spaces). The two different structures are related by a nonlinear transformation which shows how these types of transformations arise within the quantum framework as described in [37, 38.

6.2. A non trivial combination: the $f$-deformed Harmonic oscillator. Let us consider a construction similar to the previous case, but with some novelties. Let us introduce the operators

$$
A=a f(\hat{n}) \quad A^{\dagger}=f(\hat{n}) a^{\dagger},
$$

where $f$ is a certain smooth function of the number operator (or equivalently, a function of the energy of the system). As the number operator is a constant of the motion for the dynamics of the Harmonic oscillator, it is clear that the dynamics in this new operator basis has the same form:

$$
\dot{A}=-i A \quad \dot{A}^{\dagger}=i A^{\dagger}
$$


Associated with these operators we can also define a set of vector states (the vacuum state $|0\rangle$ is the same as before):

$$
|N\rangle=\frac{\left(A^{\dagger}\right)^{n}}{\sqrt{n !}}|0\rangle,
$$

which are the eigenstates of a number operator defined as

$$
\hat{N}=A^{\dagger} A \text {. }
$$

A scalar product on this new set is defined also as

$$
\langle N \mid M\rangle=\delta_{N M}
$$

We see thus that there is a one-to-one correspondence between the two sets of states, since the mapping

$$
\Pi:|k\rangle \rightarrow|K\rangle \quad k, K \in \mathbb{N}_{0}
$$

is clearly bijective. It is important to remark, though, that the linear structures are not preserved by this mapping, which is nonlinear, i.e. $\Pi\left(\left|n_{1}\right\rangle+\left|n_{2}\right\rangle\right) \neq \Pi\left(\left|n_{1}\right\rangle\right)+$ $\Pi\left(\left|n_{2}\right\rangle\right)$. We shall come back to this point in the next section.

We have thus two different Hilbert space structures associated with the initial system. We have here a situation very similar to the classical one: the "abstract" Heisenberg-Weyl algebra is realized in different manners and gives rise to algebras of operators related by an intertwining operator which is not a linear transformation.

The situation can be summarized as follows:

- With respect to the initial Hilbert space structure, we can write the commutation relation of $a$ and $a^{\dagger}$ as

$$
\left\langle n,\left[a, a^{\dagger}\right] m\right\rangle=\delta_{n m}
$$

An analogous relation exists for the operators $A$ and $A^{\dagger}$ with respect to the new Hermitian structure:

$$
\left\langle N,\left[A, A^{\dagger}\right] M\right\rangle=\delta_{N M} .
$$

- But if we consider $A$ and $A^{\dagger}$ as operators acting on the original Fock space, the commutation relations with respect to this action have the following form

$$
\left\langle n,\left[A, A^{\dagger}\right] m\right\rangle=\left((n+1) f^{2}(n+1)-n f(n)^{2}\right) \delta_{n m} .
$$

We can write thus that, with respect to the original Fock space structure,

$$
\left[A, A^{\dagger}\right]=(n+1) f^{2}(n+1)-n f(n)^{2} \mathbb{I}
$$

However the equations of motion of the Harmonic oscillator, i.e. the vector field, are the same in both cases, but the choice of one Hilbert space structure or the other makes the description quite different when written in the "wrong" framework. And notice also that both Hilbert space structures are related to each other by a transformation which is nonlinear. The corresponding transformations at the level of the symplectic structures (i.e. the transformations which relate the commutation relations) define a non-canonical nonlinear transformation.

We can give some more details of the construction if we choose a particular example of the mapping $f$ : 
6.2.1. One dimensional example. A typical example of this construction is provided by the so-called $q$-deformed Harmonic oscillators, defined by the expressions:

$$
n_{q}=\frac{\sinh n \hbar}{\sinh \hbar} \quad q=e^{\hbar}
$$

where $n$ is the undeformed number operator introduced above. Analogously the creation-annihilation operators are written in terms of the undeformed ones:

$$
a_{q}=a f(n) \quad a_{q}^{+}=f(n) a^{+}
$$

where

$$
f(n)=\sqrt{\frac{n_{q}}{n}}
$$

Notice that this transformation is invertible, since we can write

$$
a=A \sqrt{\left(\frac{\log \left(\hat{N} \sinh \hbar+\sqrt{\hat{N}^{2} \sinh ^{2} \hbar+1}\right.}{\hbar \hat{N}}\right)} .
$$

But it is simple to read from here the non-linearity of the mapping $\Pi:|k\rangle \rightarrow|K\rangle$ introduced above, since the relation for the number operator is thus written as:

$$
\hat{n}=\frac{1}{\hbar} \log \left(\hat{N} \sinh \hbar+\sqrt{\hat{N}^{2} \sinh ^{2} \hbar+1}\right) .
$$

The mapping $\Pi$ is just read from here since it is defined on the set of eigenstates of these two operators $(\hat{n}$ and $\hat{N})$.

The expression for the new commutation relation in terms of the old Hilbert space structure corresponds to

$$
\left[A, A^{\dagger}\right]=\left(\hat{N}(\cosh \hbar-1)+\sqrt{\hat{N}^{2} \sinh ^{2} \hbar+1}\right) \mathbb{I}
$$

For this scheme, we define the Hamiltonian of the $q$-deformed oscillator to be:

$$
H_{q}=\frac{1}{\hbar} \log \left(\hat{N} \sinh \hbar+\sqrt{\hat{N}^{2} \sinh ^{2} \hbar+1}+\frac{1}{2}\right) .
$$

With this operator and the original Hilbert space structure, we can write the associated vector field:

$$
\left[A, H_{q}\right]=A,
$$

which gives the original dynamics written in terms of the new operators.

6.2.2. Two dimensional case. For the cases with more than one oscillator we have the option of keeping or not the $U(N)$ symmetry of the undeformed problem. If we prefer to keep the symmetry, we define the deformed creation and annihilation operators as:

$$
\begin{array}{ll}
a_{q}=a f(n) & a_{q}^{+}=f(n) a^{+} \\
b_{q}=a f(n) & b_{q}^{+}=f(n) a^{+}
\end{array}
$$

where now $n=n_{a}+n_{b}$ (to break it, one should choose for instance only one of the number operators for each deformation).

The Hamiltonian is now obtained as:

$$
H=\frac{1}{2}\left[n f^{2}(n)+(n+2) f^{2}(n+1)\right]
$$


which exhibits the kind of combination of models we have exposed above. It is a combination of Harmonic oscillators with a function which is a "constant of the motion" on each description.

If we want to break the symmetry and define a different combination for the Hamiltonian, we define the creation and annihilation operators as:

$$
\begin{aligned}
a_{q}=a f\left(n_{a}\right) & a_{q}^{+}=f\left(n_{a}\right) a^{+} \\
b_{q}=a f\left(n_{b}\right) & b_{q}^{+}=f\left(n_{b}\right) a^{+}
\end{aligned}
$$

in such a way that the Hamiltonian becomes:

$$
H=\frac{1}{2}\left[n_{a} f^{2}\left(n_{a}\right)+n_{b} f^{2}\left(n_{b}\right)+\left(n_{a}+1\right) f^{2}\left(n_{a}+1\right)+\left(n_{b}+1\right) f^{2}\left(n_{b}+1\right)\right.
$$

In this case is even more clear the way models are combined. We have two different oscillators, and the construction of a Hamiltonian with different factor for each representation of them.

The mapping $\Phi$ relating the two descriptions, acts only on each particular representation of the $H_{4}$ algebra of each particular oscillator. It transforms the algebra and leaves invariant the Hilbert space, which is constructed as the product of both Fock spaces in the usual way. The global transformation is constructed as:

$$
\Phi=\sum f\left(n_{i}\right) \Phi^{i}
$$

where $\Phi^{i}$ is the one dimensional transformation and it is equal to the identity.

6.3. The Coulomb problem. As an example of the potential applications of the composition of models to the study of quantum problems let us consider a simple example in the Schrödinger picture.

It is well known (see for instance [20]) that the system may be described with the help of a combination of Harmonic oscillators for the bound states in the $D=2$ and $D=3$ cases. From our point of view this implies that the system is integrable at the quantum level (for these dimensions and regimes).

For both cases we decompose the Hilbert space in the eigenspaces of the Hamiltonian, let us call them $U_{E_{\alpha}}$. Now, on each subspace we perform a change of coordinates that maps $U_{E_{\alpha}}$ on a suitable subspace of a Fock space.:

- For the two dimensional case the change of coordinates is simply the transformation into parabolic cylinder coordinates:

$$
x=\frac{1}{2}\left(u^{2}-v^{2}\right) \quad y=u v
$$

where the point $(-u,-v)$ and $(u, v)$ are identified. This change transform the eigenvalue equation

into

$$
-\frac{1}{2}\left(\frac{\partial^{2} \psi}{\partial x^{2}}+\frac{\partial^{2} \psi}{\partial y^{2}}\right)-\frac{k}{\sqrt{x^{2}+y^{2}}} \psi=E \psi
$$

$$
-\frac{1}{2}\left(\frac{\partial^{2} \psi}{\partial u^{2}}+\frac{\partial^{2} \psi}{\partial v^{2}}\right)-\frac{1}{2} \omega^{2}\left(u^{2}+v^{2}\right) \psi=E^{\prime} \psi
$$

where the wave-functions should verify $\psi(-u,-v)=\psi(u, v)$, where $\omega^{2}=$ $2|E|=\frac{2 k}{n^{2}}$ and $E^{\prime}=k=n \omega$ where $n$ has to be odd to satisfy the parity condition of the wave functions. We construct thus a mapping from the Hilbert space of bound states of the two dimensional Coulomb problem 
into the subspace of the product Fock space $V_{u} \otimes V_{v}$ corresponding to an odd number of particles. There is a dependence of the mapping on the Hamiltonian, which produces a different frequency for each oscillator.

$$
\Phi_{E_{\alpha}}: U_{E_{\alpha}} \rightarrow V_{u v}^{o d d} \subset V_{u} \otimes V_{v}
$$

The complete mapping is the union of these $\Phi_{E}$ which are formally identical:

$$
\Phi=\cup_{\alpha} \Phi_{E_{\alpha}}
$$

- For the three dimensional case, the situation is analogous (see [20] as the original reference) . Now we make use of the $\mathfrak{s u}(2) \oplus \mathfrak{s u}(2)$ symmetry algebra of the Coulomb problem (corresponding to suitable combinations of the angular momentum and the Runge-Lenz vector if we define it in terms of generators). We construct then a two dimensional Harmonic oscillator associated with each of the $\mathfrak{s u}(2)$ group, thus obtaining a four dimensional isotropic Harmonic oscillator.

The change of coordinates is constructed in two steps:

$$
\begin{array}{r}
x_{1}=\mu \nu \cos \phi \quad x_{2}=\mu \nu \sin \phi \quad x_{3}=\frac{1}{2}\left(\mu^{2}-\nu^{2}\right) \\
\xi_{\mu}=\mu \cos \phi \quad \eta_{\mu}=\mu \sin \phi \\
\xi_{\nu}=\nu \cos \phi \quad \eta_{\nu}=\nu \sin \phi
\end{array}
$$

where again $\omega^{2}=2|E|$ (the change is different for each eigenspace of the Hamiltonian). These changes transform now the system (this time considered in three dimensions) into:

$$
H \psi=\left(H_{\mu}+H_{\nu}\right) \psi=E^{\prime} \psi
$$

where

$$
H_{\alpha}=-\frac{1}{2}\left(\frac{\partial^{2}}{\partial \xi_{\alpha}^{2}}+\frac{\partial^{2}}{\partial \eta_{\alpha}^{2}}\right)-\frac{1}{2} \omega^{2}\left(\xi_{\alpha}^{2}+\eta_{\alpha}^{2}\right)
$$

and $E^{\prime}=k=\omega\left(n_{\mu}+n_{\nu}+1\right)$.

The mapping should be understood now applying each eigenspace of the Hamiltonian of the hydrogen atom, representation by representation of the angular momentum and the Runge-Lenz vector, onto the corresponding representations of the Hamiltonian of the Harmonic oscillator.

We can write analogously to the previous case:

$$
\Phi_{E_{\alpha}}: U_{E_{\alpha}} \rightarrow V_{\mu \nu}^{o d d} \subset V_{\mu} \otimes V_{\nu}
$$

where now $V_{\alpha}$ is the Fock space corresponding to a two dimensional oscillator. For the whole Hilbert space we can write again:

$$
\Phi=\cup_{\alpha} \Phi_{E_{\alpha}}
$$

A different approach, where starting from the classical situation the construction of the quantum one is directly related to the geometry of the Lagrangian description is provided in [5]. 


\section{Conclusions And outlook}

In this paper we have pointed out various problems that a definition of quantum integrability has to face. The main one seems to be the definition of functional independence which, in our present understanding, requires a differential calculus on commutative algebras. This calculus deals with derivations and is fully captured by the notion of differential manifold, be it finite or infinite dimensional. For this reason we believe that the geometrization of quantum mechanics can be a good starting point. In this approach we find that alternative products on functions coexist:

- a non-local product (on quadratic functions) which captures the essence of quantum mechanics in terms of indetermination relations and a rule for composing systems,

- a local commutative product which allows for a differential calculus in terms of derivations which are local.

At the moment, it seems that the latter is useful, if not unavoidable, to state functional independence of "observations" (quadratic function version of the observables).

Usual treatments of integrability $([11,49])$ at the quantum level deal with Hilbert spaces considered as spaces of square integrable functions on some "configuration space". It seems that the notions we are advocating are provided by Lie groups and their unitary representations, along with the associated representations of their Lie algebras (and their enveloping algebras) on the set of analytic or smooth vectors [48, 16].

The convolution product and the pointwise product available on the group seem to be related to the two products we are identifying. All these considerations appear very neatly in the so called Quantum Mechanics on phase space (the WignerWeyl formalism, see [8]) There the phase space is an Abelian vector group and the convolution product (Moyal product) is associated with its central extension defined with the help of a symplectic structure). The algebra of differential operators is identified as the algebra of operators acting on square integrable functions defined on Lagrangian subspaces of this symplectic vector space.

The representation of the enveloping algebra allows us to deal with suitable deformations (quantum groups and Yang Baxter relations).

The transformation from one Lagrangian subspace to another one requires the introduction of pseudo-differential operators. Nonlinearly related actions of $\mathbb{R}^{2 n}$ on the phase space, when considered as Weyl systems [21, give rise to nonlinear transformations in the quantum framework.

Some of these statements need further elaboration to be able to convert them into propositions and theorems. We hope to come back to these questions in the near future.

Another spin-off of this geometrization procedure is the possibility of using methods and conceptual constructions elaborated in the framework of classical physics (vector fields, Poisson brackets, Riemannian vector fields) also in the framework of quantum physics, with the additional intervention of additional structures which may be traced back to the existence of the fundamental constant of nature provided by Planck's constant [4]. 
The difficulties we have to face can be traced back to the lack of a mature mathematical treatment of infinite-dimensional differential manifold even though very good books are already available $([33,10,30])$. Our hope is to be able to apply in the very near future these methods to study quantum control problems using as much as possible the experience available in the "classical framework".

Acknowledgements This paper is an extended version of the seminar given by one of us (J. C-G) in the framework of the Conference "Mathematical Structure on Quantum Mechanics II" which took place in Madrid (Spain), in March 2008. We would like to thank the organizers of that event for giving us the opportunity of presenting the work and to the participants for their interesting comments and suggestions.

\section{REFERENCES}

[1] R Abraham and J. E. Marsden. Foundation of Mechanics. Benjamin, 1978.

[2] D. Alekseevsky, J. Grabowski, G. Marmo, and P.W. Michor. Completely integrable systems: a generalization. Mod Phys Lett A, 12:1637-1648, 1997.

[3] V. I. Arnold. Mathematical methods of classical mechanics. Springer, 1989.

[4] A. D' Avanzo and G. Marmo. Reduction and unfolding: the Kepler problem. Int. J. Geom. Meth. Phys., 2:83-109, 2005.

[5] A. D' Avanzo, G. Marmo, and A. Valentino. Reduction and unfolding for quantum systems: the Hydrogen atom. Int. J. Geom. Meth. Phys, 2:1043-1062, 2005.

[6] G. Bimonte, G. Marmo, and A. Stern. Bicovariant calculus in quantum theory and a generalization of the Gauss law. Phys Lett B, 478:358, 2000.

[7] J. F. Cariñena, J. Clemente-Gallardo, and G. Marmo. Geometrization of Quantum Mechanics. Theor. Math Phys, 152(1):894-903, 2007.

[8] J. F. Cariñena, J. Clemente-Gallardo, and G. Marmo. Proceedings of the XV International Workshop on Geometry and Physics, volume 11 of Publicaciones de la RSME, chapter Introduction to Quantum Mechanics and the Quantum-Classical transition, pages 3-45. RSME, 2007.

[9] J. F. Cariñena, J. Grabowski, and G. Marmo. Lie-Scheffers systems: a geometric approach. Bibliopolis, Napoli, 2000.

[10] H. Cartan. Differential forms (trans. from french). Houghton Mifflin Co, Boston, 1970.

[11] G. Cassinelli, E. de Vito, and A. Levrero. Integrability of the quantum adiabatic evolution and geometric phases. J. Math. Phys., 38(12):6101-6118, 1997.

[12] R. Cirelli, A. Maniá, and L. Pizzocchero. A functional representation for noncommutative $\mathbb{C}^{*}$-algebras. Rev Math Phys, 6(5):675-697, 1994.

[13] J. Clemente-Gallardo and G. Marmo. Differential Geometric Methods in Mechanics and Field Theory, chapter The space of density states in geometrical quantum mechanics, pages 35-56. Gent Academia Press, 2007.

[14] J. Clemente-Gallardo and G. Marmo. Basics of quantum mechanics, geometrization and some applications to quantum information. Int. J. Geom. Meth. Mod. Phys, 5(6):-, 2008.

[15] A. Connes. Noncommutative Geomtry. Academic Press, 1994.

[16] E. B. Davies. Hilbert space representations of Lie algebras. Comm. Math. Phys., 23:159-168, 1971.

[17] J. Dixmier. Les $\mathbb{C}^{*}$-algèbres et leurs réprésentations. Gauthiers-Villars, Paris, 1964.

[18] B. Dubrovin, G. Marmo, and A. Simoni. Alternative hamiltonian description for quantum systems. Modern Physics Letters A, 15(5):1229-1234, 1990.

[19] G. G. Emch. Foundations of 20th century Physics. North Holland, 1984.

[20] M.J. Engeldfeld. Group theory and the Coulomb problem. Wiley-Interscience, 1972.

[21] E. Ercolessi, A. Ibort, G. Marmo, and G. Morandi. Alternative linear structures for classical and quantum systems. Int. J. Mod. Phys. A, 22(18):3039-3064, 2007.

[22] L. D. Faddeev. Nonlinear equations and spectral theory, chapter What is complete integrability in quantum mechanics. AMS Trans. Serv 2, 220, Providence, 2007.

[23] L. D. Faddeev and L. A. Takhtajan. Hamiltonian methods in the theory of solitons. Springer, Berlin, 2007. 
[24] S. De Filippo, G. Vilasi, and G. Marmo. A new characterization of completely integrable systems. Nuovo Cimento B, 83(2):97-112, 1984.

[25] I. M. Gelfand and I. Ya. Dorfman. Hamiltonian operators and the classical yang-baxter equation (russian). Function. Anal. Appl., 16(4):241-248, 1982.

[26] Janusz Grabowski, Marek Kus, and Giuseppe Marmo. Geometry of quantum systems: density states and entanglement. J.Phys. A, 38:10217-10244, 2005.

[27] Janusz Grabowski, Marek Kus, and Giuseppe Marmo. Symmetries, group actions, and entanglement. Open Syst. Inf. Dyn., 13:343-362, 2006.

[28] J. Hietarinta. Classical versus quantum integrability. J. Math. Phys., 25:1833, 1989.

[29] A. A. Kirillov. Merits and demerits of the orbit method. Bull. AMS, 36:433-488, 1999.

[30] I. Kolar, P. W. Michor, and J. Slovak. Natural operations in differential geometry. SpringerVerlag, Berlin, 1993.

[31] G. Landi. An introduction to noncommutative spaces and their geometries, volume 51 of Lecture Notes in Physics. Springer-Verlag, Berlin, 1997.

[32] N. P. Landsman. Mathematical topics between Classical and Quantum Mechanics. SpringerVerlag, 1998.

[33] S. Lang. Introduction to differentiable manifolds. Spinger-Verlag, New York, 2002.

[34] P. D. Lax. Integrals of nonlinear equations of evolution and solitary waves. Comm. Pure Appl. Math, 21:467-490, 1968.

[35] S. Lie and G. Scheffers. Vorlesungen über continuierliche Gruppen mit geometrischen und anderen Anwendungen. Teubner, Leipzig, 1893. Edited and revised by G. Scheffers.

[36] F. Magri. A simple model of the integrable hamiltonian equation. J. Math. Phys., 19(5):1156$1162,1978$.

[37] V. I. Manko, G. Marmo, E.C.G. Sudarshan, and F. Zaccaria. $f$-oscillators and nonlinear coherent states. Physica Scripta, 55:528-541, 1997.

[38] V. I. Manko, G. Marmo, F. Zaccaria, and E.C.G. Sudarshan. Wigner's problem and alternative commutation relations for quantum mechanics. Int. J. Modern Physics B, 11(10):1281$1296,1997$.

[39] V.I. Man'ko, G. Marmo, S. Solimeno, and F. Zaccaria. Physical non linear aspects of classical and quantum q-oscillators. Int. Jour. Mod. Phys. A, 8(20):3577-3597, 1993.

[40] G. Marmo, G. Morandi, A. Simoni, and F. Ventriglia. Alternative structures and bihamiltonian systems. J. Phys A: Math Gen, 35:8393-8406, 2002.

[41] G. Marmo, E. J. Saletan, A. Simoni, and B. Vitale. Dynamical Systems, a differential geometric approach to symmetry and reduction. John Wiley, Chicester, 1985.

[42] G. Marmo, G. Scolarici, A. Simoni, and F. Ventriglia. Alternative structures and biHamiltonian systems on a Hilbert space. J. Phys. A: Math and Gen, 38(17):3813-3821, 2005.

[43] G. Marmo, G. Scolarici, A. Simoni, and F Ventriglia. The quantum-classical transition: the fate of the complex structure. Int. J. Geom. Meth. Mod. Phys, 2:127-145, 2005.

[44] G. Marmo and G. Vilasi. When do recursion operators generate new conservation laws? Phys Lett B, 277:137-140, 1992.

[45] G. Marmo, P. Vitale, and A. Zampini. Noncommutative differential calculus for Moyal subalgebras. J. Geom. Phys., 55:611-622, 2006.

[46] P. W. Michor and T. S. Ratiu. On the geometry of the Virasoro-Bott group. J. Lie Theory, 8(2):293-309, 1998.

[47] G. Morandi, C. Ferrario adn G. Lo Vecchio, G. Marmo, and C. Rubano. The inverse problem in the calculus of variations and the geometry of the tangent bundle. Phys. Rep., 188(34):147-284, 1990.

[48] E. Nelson. Analytic vectors. Ann. Math., 70:572-615, 1959.

[49] M. B. Scheftel, P. Tempesta, and P. Winternitz. Superintegrable systems in quantum mechanics and classical lie theory. J. Math. Phys, 42(2):659-673, 2001.

[50] I. E. Segal. Quantized differential forms. Topology, 8:147, 1967.

[51] I. E. Segal. Quantization of the de Rham complex. Proc. Symp. Pure Math, (16):205, 1970.

[52] R. F. Streater. The representation of the oscillator group. Comm. Math. Phys., 4:217-236, 1967.

[53] V. S. Varadarajan. An introduction to harmonic analysis on semisimple Lie groups. Cambridge studies in Advanced Mathematics. Cambrigde University Press, 1989.

[54] G. Vilasi. Hamiltonian dynamics. World Scientific, River Edge, NY, 2001. 
[55] J. von Neumann. Mathematical Foundations of Quantum Mechanics. Princeton Univ. Press, 1996.

[56] S. Weigert. The problem of quantum integrability. Physica D, 56(1):107-119, 1992.

Instituto de Biocomputación y Física de los Sistemas Complejos, Universidad de Zaragoza, Corona de Aragón 42, 50009 Zaragoza (SPAIN), and, Departamento de Física Teórica, Facultad de Ciencias, Universidad de Zaragoza, 50009 Zaragoza (SPAIN),

E-mail address: jcg@unizar.es

Dipartamento di Física Teorica, Universitá Federico II and INFN Sezione di Napoli, Via CinTIA, 80126 NAPOLI (ITALY)

E-mail address: marmo@na.infn.it 